\title{
Casimir energy and topological mass for a massive scalar field with Lorentz violation
}

\author{
M. B. Cruz $\oplus^{1, *}$ E. R. Bezerra de Mello๑, ${ }^{2, \dagger}$ and H. F. Santana Mota $\oplus^{2, \$}$ \\ ${ }^{1}$ Departamento de Física, Universidade Federal de Campina Grande, Caixa Postal 882, \\ 58428-830 Campina Grande, Paraíba, Brazil \\ ${ }^{2}$ Departamento de Física, Universidade Federal da Paraíba, Caixa Postal 5008, \\ 58051-970 João Pessoa, Paraíba, Brazil
}

(Received 23 May 2020; revised 19 July 2020; accepted 23 July 2020; published 6 August 2020)

\begin{abstract}
A Lorentz symmetry violation aether-type theoretical model is considered to investigate the Casimir effect and the generation of topological mass associated with self-interacting massive scalar fields obeying Dirichlet, Newman, and mixed boundary conditions on two large and parallel plates. By adopting the path integral approach, we find the effective potential at one- and two-loop corrections, which provides both the energy density and the topological mass when taken in the ground state of the scalar field. We then analyze how these quantities are affected by the Lorentz symmetry violation and compare the results with previous ones found in literature.
\end{abstract}

DOI: $10.1103 /$ PhysRevD.102.045006

\section{INTRODUCTION}

The Casimir effect was predicted by H. B. Casimir in 1948 [1] and, although not with great precision, experimentally verified ten years later by M. J. Sparnaay [2]. Since then, it has been confirmed by several highprecision experiments [3-9], leading currently to one of the most interesting topics of research. The Casimir effect consists of a direct manifestation of the existence of quantum fluctuations of the vacuum and was noted to arise for the first time when considering two parallel conducting plates placed very close to each other in the vacuum, separated by a very small distance when compared with the plates' dimensions. In this case, the theoretical prediction and experimental observation that the two plates attract each other [1] was not credited to the gravitational or electromagnetic forces, but to modifications of the quantum vacuum fluctuations of the electromagnetic field by the presence of the plates. The gravitational interaction between the plates is far too weak to be observed, while the electromagnetic interaction is absent, since the plates are neutral.

\footnotetext{
*messiasdebritocruz@gmail.com †emello@fisica.ufpb.br

thmota@fisica.ufpb.br
}

Published by the American Physical Society under the terms of the Creative Commons Attribution 4.0 International license. Further distribution of this work must maintain attribution to the author(s) and the published article's title, journal citation, and DOI. Funded by SCOAP.
Other quantum-relativistic fields, such as scalar and fermion fields, can also present modifications in the quantum fluctuations of their vacua by some sort of boundary condition, leading to a Casimir-like effect. The formal and standard approach to investigating the Casimir interactions is in the realm of quantum field theory, which is based on the assumption that the Lorentz symmetry is preserved. However, one may well assume other scenarios where the Lorentz symmetry is violated, which is normally the case in models that look for probing high-energy phenomena. This, in fact, has been done from both theoretical and experimental points of view. In the context of several of these Lorentz symmetry violation scenarios, the spacetime becomes anisotropic in one (or more) direction, including time, and inevitably the quantum field whose modes propagate in it has its energy spectrum modified. Lorentz symmetry violation in string theory can be found in Ref. [10] and in low-energy scale scenarios in Refs. [11-19]. In these contexts, Casimir energy has been considered in Refs. [20,21] and [22-24], respectively. Therefore, with such a great number of theoretical works, it is natural that the search for Lorentz symmetry violations also acquire experimental interest and, in this sense, the Casimir effect becomes an even more interesting topic to study, since it can be related with Lorentz symmetry violation models.

Although the Casimir effect is more often calculated in terms of the zero-point energy of a quantized field, this effect can also be investigated by adopting the path integral formalism for quantum field theory, developed by Jackiw [25], in which an effective potential, presented in terms of loop expansions, allows us to obtain the energy density 
as well as the generation of topological mass. ${ }^{1}$ Studies of radiative corrections for the Casimir energy were reported in Refs. [26-28] and in Refs. [29-31]. In the latter, the generation of topological mass for a self-interacting massless scalar field obeying different boundary conditions on two large and parallel plates was also considered. By following the same line of investigation as in Ref. [30], in the present work, we study the loop expansions to the Casimir energy and generation of topological mass for selfinteracting massive and massless scalar fields subject to Dirichlet, Newman, and mixed boundary conditions in the context of a $C P T$-even aether-type Lorentz symmetry violation model [32-34].

This paper is organized as follows: In Sec. II, we briefly describe the theoretical model that we want to investigate, which consists of a self-interacting massive scalar field in a $C P T$-even aether-type Lorentz symmetry violation approach. We then calculate the one- and two-loop radiative corrections to the Casimir energy and the generation of topological mass, admitting that the scalar field obeys Dirichlet, Newman, and mixed boundary conditions on two large and parallel plates. Because these calculations are divergent, we adopt the Riemann zeta-function renormalization procedure to provide finite and well-defined results. Finally, in Sec. III, we present our conclusions. Throughout the paper, we use natural units $\hbar=c=1$ and the metric signature $(-,+,+,+)$.

\section{LOOPS CORRECTIONS AND GENERATION OF TOPOLOGICAL MASS}

\section{A. Theoretical model}

We first introduce the aether-type Lorentz symmetry violation model that we want to consider to investigate the vacuum energy and generation of topological mass. The model is composed of a self-interacting scalar field that presents a $C P T$-even and aether-like Lorentz violation term implemented by direct coupling between the derivative of the field with an external constant four-vector. (For a more detailed review, see Refs. [32,34]). The model is described by the action below:

$$
\mathcal{S}(\phi)=\int_{\mathcal{M}} d^{4} x \mathcal{L}(x),
$$

where $\mathcal{M}$ is a flat manifold and $\mathcal{L}$ is a Lagrangian density, given by

$$
\mathcal{L}(x)=-\frac{1}{2}\left(\partial_{\mu} \phi\right)\left(\partial^{\mu} \phi\right)+\frac{1}{2} \chi(u \cdot \partial \phi)^{2}-U(\phi) .
$$

In the above expression, the scalar field of mass $m$ is represented by $\phi(x)$. The four-vector, $u^{\mu}$, is responsible for

\footnotetext{
${ }^{1}$ In fact, both calculations are divergent. The standard procedure to renormalize them and obtain finite results is through the use of the Riemann zeta function.
}

a privileged direction in spacetime; and the dimensionless parameter $\chi$, which codifies the Lorentz violation, is much smaller than unity. The last term on the rhs of Eq. (2.2) is the classical potential $U(\phi)$, which, for a massive and selfcoupling $\lambda \phi^{4}$ theory, is given by

$$
U(\phi)=\frac{m^{2} \phi^{2}}{2}+\frac{\lambda \phi^{4}}{4 !}+\frac{\phi^{4}}{4 !} \delta_{1}+\frac{\phi^{2}}{2} \delta_{2}+\delta_{3},
$$

where the parameters $\delta_{1}, \delta_{2}$, and $\delta_{3}$ correspond to the renormalization constants of the theory and will be determined later. Before we proceed, we want to make clear that the analysis we want to develop in this paper will take into consideration the four-vector, $u^{\mu}$, in two types: timelike and spacelike. The timelike component is represented by $u^{t}=(1,0,0,0)$; while the spacelike is represented by $u^{x}=$ $(0,1,0,0)$ if the privileged direction is in the $x$ axis, $u^{y}=$ $(0,0,1,0)$ if the privileged direction is in the $y$ axis, and $u^{z}=(0,0,0,1)$ if the privileged direction is in the $z$ axis.

In order to adopt the path integral approach described in detail in Ref. [30], we need to allow the field $\phi(x)$ to fluctuate about a fixed background field, $\Phi$, with its quantum fluctuations represented by $\varphi$. Thus, after performing a Wick rotation $(t \rightarrow-i t)$ in the Lorentzian action [Eq. (2.1)] and defining a Euclidean one, we can make use of the generating function of the one-particle-irreducible Green function [30]. This provides a description in terms of a $\Phi$-dependent effective potential which, up to two-loop corrections, is written as

$$
V_{\mathrm{eff}}(\Phi)=V_{\mathrm{cl}}(\Phi)+V^{(1)}(\Phi)+V^{(2)}(\Phi)
$$

where $V_{\mathrm{cl}}(\Phi)=U(\Phi)$ is the tree-level (classical) contribution to the effective potential in a flat manifold, and $V^{(1)}(\Phi)$ and $V^{(2)}(\Phi)$ are the one- and two-loop correction contributions, respectively. Note that we have performed a linear expansion of $\phi(\phi \rightarrow \Phi+\varphi)$ about the classical field, $\Phi$. The two-loop contribution in the last term on the rhs of Eq. (2.4) is a contribution of two graphs to the effective Euclidian action [30]. We will postpone to the next sections how to calculate it for each case we consider.

As to the one-loop contribution to the effective potential, we will follow the same method as Ref. [30], which is to define this contribution in terms of the Riemann zeta function $\zeta(s)$, i.e.,

$$
V^{(1)}(\Phi)=-\frac{1}{2 \operatorname{vol}(\mathrm{E})}\left[\zeta^{\prime}(0)+\zeta(0) \ln \left(\mu^{2}\right)\right],
$$

where $\operatorname{vol}(\mathrm{E})$ is the Euclidian volume, $\zeta^{\prime}(s)$ the derivative of the zeta function with respect to the parameter $s$, and the term $\zeta(0) \ln \left(\mu^{2}\right)$ is to be removed by renormalization. ${ }^{2}$

\footnotetext{
${ }^{2}$ The parameter $\mu$ is associated with a measure on the space of function [30].
} 
As is known, the (generalized) Riemann zeta function, $\zeta(s)$, is defined as

$$
\zeta(s)=\sum_{\beta} \Lambda_{\beta}^{-s},
$$

where $\Lambda_{\beta}$ is the spectrum of eigenvalues associated with a self-adjoint elliptic operator, which in our case is given by

$$
\Delta=-\partial_{\mu} \partial^{\mu}+\chi u^{\mu} u^{\nu} \partial_{\mu} \partial_{\nu}+m^{2}+\frac{\lambda \Phi^{2}}{2} .
$$

Note that $\beta$ stands for the set of quantum numbers associated with the quantum field eigenfunction, $\varphi$, of the operator, $\Delta$. Although the zeta function (2.6) is defined in terms of the complex parameter $s$, for $\operatorname{Re}(s)>1$, an analytic continuation to the whole complex plane can be obtained for it, including in $s=0$.

The renormalization condition that enables us to eliminate the term $\zeta(0) \ln \left(\mu^{2}\right)$ in Eq. (2.5) is considered in analogy to Coleman-Weinberg and should fix the coupling constant $[30,35]$. This condition is written as

$$
\left.\frac{d^{4} V_{\text {eff }}}{d \Phi^{4}}\right|_{\Phi=0}=\lambda
$$

As we will see, this condition will fix the renormalization constant $\delta_{1}$.

On the other hand, the condition that makes it possible to obtain a topological mass is given by

$$
\left.\frac{d^{2} V_{\mathrm{eff}}}{d \Phi^{2}}\right|_{\Phi=0}=m^{2}
$$

This condition fixes the renormalization constant $\delta_{2}$ and also provides the topological mass when using the renormalized effective potential, as we will see. Note that $\Phi=0$ is the value that minimizes the effective potential and represents the minimum of the potential only if it obeys the extremum condition

$$
\left.\frac{d V_{\mathrm{eff}}}{d \Phi}\right|_{\Phi=0}=0
$$

leading to Eq. (2.9) being positive.

Moreover, in order to find the constant $\delta_{3}$, we also need to use an additional renormalization condition, which is given by

$$
\left.V_{\text {eff }}\right|_{\Phi=0}=0
$$

From now on in our discussion, we will assume that the quantum field $\varphi$ is confined between two large parallel plates, as shown in Fig. 1. The quantum field $\varphi$ is an eigenfunction of the self-adjoint elliptic operator [Eq. (2.7)], with eigenvalues $\Lambda_{\beta}$. In this sense, the

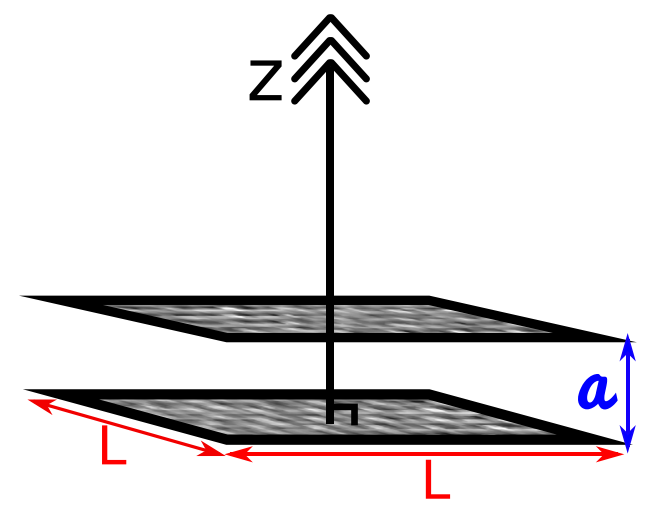

FIG. 1. Schematic configuration for the two parallel plates with area $L^{2}$ separated by a distance $a(a \ll L)$.

eigenvalues we are interested in are the ones obtained by requiring that $\varphi$ must satisfy specific boundary conditions on the plates placed at $z=0$ and $z=a$ for all cases of four-vector $u^{\mu}$ : timelike and spacelike.

\section{B. Dirichlet and Neumann boundary conditions}

We will start by considering the case where the scalar field, $\varphi$, satisfies Dirichlet and Neumann boundary conditions on the plates-that is,

$$
\begin{aligned}
\left.\varphi(x)\right|_{z=0} & =\left.\varphi(x)\right|_{z=a}, \quad \text { and } \\
\left.\frac{\partial \varphi(x)}{\partial z}\right|_{z=0} & =\left.\frac{\partial \varphi(x)}{\partial z}\right|_{z=a}, \quad \text { respectively. }
\end{aligned}
$$

The complete set of normalized solutions of the scalar field, $\varphi$, under these conditions has been reported, for instance, in Ref. [36]. These solutions provide the following eigenvalues of the operator (2.7):

$\Lambda_{\beta}=k_{t}^{2}+k^{2}+\frac{n^{2} \pi^{2}}{a^{2}}-\chi u^{\mu} u^{\nu} k_{\mu} k_{\nu}+m^{2}+\frac{\lambda \Phi^{2}}{2}$,

where $k_{\mu}=\left(k_{t}, k_{x}, k_{y}, k_{z}\right)$ are the four-momentum components, $k^{2}=k_{x}^{2}+k_{y}^{2}$ and $k_{z}=n \pi / a$, for $n=1,2,3, \ldots$ Hence, the set of quantum numbers is $\beta=\left(k_{t}, k_{x}, k_{y}, n\right)$. Note that $k_{t}, k_{x}$, and $k_{y}$ are continuum quantum numbers. In addition, we want to point out that for a constant timelike vector, $u^{t}$, in Eq. (2.13), we have to consider the Euclidean version of the zero component of the four-momentum; i.e., we have to take $k_{t} \rightarrow-i k_{t}$, in the term associated with the Lorentz-violating parameter, $\chi$.

\section{Timelike vector}

We want to begin considering the timelike type of the four-vector $u^{\mu}$, in which case $u^{t}=(1,0,0,0)$, meaning that the privileged direction chosen to have the Lorentz symmetry violated is the time one. This leads the eigenvalues (2.13) to be written as 


$$
\Lambda_{\beta}=(1+\chi) k_{t}^{2}+k^{2}+\frac{\pi^{2} n^{2}}{a^{2}}+m^{2}+\frac{\lambda \Phi^{2}}{2} .
$$

The set of eigenvalues in Eq. (2.14) allows us to build the zeta function by using the definition (2.6). This provides

$$
\begin{aligned}
\zeta(s)= & \frac{V_{3}}{(2 \pi)^{3}} \sum_{n=1}^{\infty} \int d^{3} k \\
& \times\left[(1+\chi) k_{t}^{2}+k^{2}+\frac{\pi^{2} n^{2}}{a^{2}}+m^{2}+\frac{\lambda}{2} \Phi^{2}\right]^{-s},
\end{aligned}
$$

where $V_{3}$ is a continuum volume associated with the coordinates $t, x, y$ and $d^{3} k=d k_{t} d k_{x} d k_{y}$. After defining a new variable $\kappa_{t}=\sqrt{1+\chi} k_{t}$, the integrals in $\kappa_{t}, k_{x}$, and $k_{y}$ can be performed by using the identity

$$
\frac{1}{\omega^{2 s}}=\frac{1}{\Gamma(s)} \int_{0}^{\infty} d \tau \tau^{2 s-1} e^{-\omega^{2} \tau^{2}} .
$$

Consequently, Eq. (2.15) becomes

$$
\zeta(s)=\frac{V_{3}}{(2 \pi)^{3}} \frac{\pi^{3 / 2}}{\sqrt{1+\chi}} \frac{\Gamma(s-3 / 2)}{\Gamma(s)} w^{3-2 s} \sum_{n=1}^{\infty}\left(n^{2}+\nu^{2}\right)^{3 / 2-s},
$$

where

$$
\nu^{2} \equiv \frac{\lambda \Phi^{2}}{2 w^{2}}+\frac{m^{2}}{w^{2}} \quad \text { and } \quad w \equiv \frac{\pi}{a} .
$$

The sum in $n$ present in Eq. (2.17) can be worked out by making use of the Epstein-Hurwitz zeta function [37]:

$$
\begin{aligned}
\zeta_{\mathrm{EH}}(s, \nu) \equiv & \sum_{n=1}^{\infty}\left(n^{2}+\nu^{2}\right)^{-s} \\
= & -\frac{\nu^{-2 s}}{2}+\frac{\pi^{1 / 2}}{2} \frac{\Gamma(s-1 / 2)}{\Gamma(s)} \nu^{1-2 s} \\
& +\frac{2^{1-s}(2 \pi)^{2 s-1 / 2}}{\Gamma(s)} \sum_{n=1}^{\infty} n^{2 s-1} f_{(s-1 / 2)}(2 \pi n \nu),
\end{aligned}
$$

where the function $f_{\gamma}(x)$ is defined in terms of the modified Bessel functions [37], $K_{\gamma}(x)$, by the following relation:

$$
f_{\gamma}(x) \equiv \frac{K_{\gamma}(x)}{x^{\gamma}} .
$$

Thus, using Eq. (2.19) in Eq. (2.17), we get

$$
\begin{aligned}
\zeta(s)= & \frac{V_{3}}{(2 \pi)^{3}} \frac{1}{\sqrt{1+\chi}}\left[-\frac{\pi^{3 / 2} w^{-2 \bar{s}} \nu^{-2 \bar{s}}}{2} \frac{\Gamma(\bar{s})}{\Gamma(\bar{s}+3 / 2)}+\frac{\pi^{1 / 2} \pi^{3 / 2} w^{-2 \bar{s}}}{2} \frac{\Gamma(\bar{s}-1 / 2)}{\Gamma(\bar{s}+3 / 2)} \nu^{1-2 \bar{s}}\right. \\
& \left.+\frac{2^{1-\bar{s}}(2 \pi)^{2 \bar{s}-1 / 2} \pi^{3 / 2} w^{-2 \bar{s}}}{\Gamma(\bar{s}+3 / 2)} \sum_{n=1}^{\infty} n^{2 \bar{s}-1} f_{(\bar{s}-1 / 2)}(2 \pi n \nu)\right]
\end{aligned}
$$

where $\bar{s}=s-3 / 2$. We should note that in order to obtain the one-loop correction to the effective potential, we have to take the limit, $s \rightarrow 0$, which means $\bar{s} \rightarrow-3 / 2$. Consequently, Eq. (2.21) provides

$$
\zeta(0)=\frac{V_{3} a}{2(2 \pi)^{4}} \frac{\pi^{2} b^{4}}{\sqrt{1+\chi}}
$$

and

$$
\zeta^{\prime}(0)=\frac{V_{3} a}{(2 \pi)^{3}}\left[-\frac{2 \pi^{2}}{3} \frac{b^{3}}{\sqrt{1+\chi} a}+\frac{3}{8} \frac{\pi b^{4}}{\sqrt{1+\chi}}-\frac{\pi}{2} \frac{b^{4} \ln (b)}{\sqrt{1+\chi}}+\frac{2 \pi b^{2}}{\sqrt{1+\chi} a^{2}} \sum_{n=1}^{\infty} \frac{K_{2}(2 b a n)}{n^{2}}\right],
$$

where the parameter $b$ is defined as

$$
b \equiv \sqrt{\frac{\lambda \Phi^{2}}{2}+m^{2}} .
$$

Hence, substituting the results in Eqs. (2.22) and (2.23) into Eq. (2.5), we find the one-loop correction to effective potential; that is,

$$
V^{(1)}(\Phi)=\frac{b^{3}}{24 \pi \sqrt{1+\chi} a}-\frac{3 b^{4}}{128 \pi^{2} \sqrt{1+\chi}}+\frac{b^{4} \ln \left(\frac{b^{2}}{\mu^{2}}\right)}{64 \pi^{2} \sqrt{1+\chi}}-\frac{b^{2}}{8 \pi^{2} \sqrt{1+\chi} a^{2}} \sum_{n=1}^{\infty} \frac{K_{2}(2 b a n)}{n^{2}} .
$$


This allows us to write the effective potential (2.4) up to one-loop correction as

$$
\begin{aligned}
V_{\mathrm{eff}}(\Phi)= & \frac{m^{2} \Phi^{2}}{2}+\frac{\lambda \Phi^{4}}{4 !}+\frac{\Phi^{2}}{2} \delta_{2}+\frac{\Phi^{4}}{4 !} \delta_{1}+\delta_{3}+\frac{b^{3}}{24 \pi \sqrt{1+\chi} a}-\frac{3 b^{4}}{128 \pi^{2} \sqrt{1+\chi}} \\
& +\frac{b^{4} \ln \left(\frac{b^{2}}{\mu^{2}}\right)}{64 \pi^{2} \sqrt{1+\chi}}-\frac{b^{2}}{8 \pi^{2} \sqrt{1+\chi} a^{2}} \sum_{n=1}^{\infty} \frac{K_{2}(2 b a n)}{n^{2}} .
\end{aligned}
$$

The effective potential above still needs to be renormalized, requiring that we find the renormalization constants $\delta_{1}, \delta_{2}$, and $\delta_{3}$ as we take the limit $a \rightarrow+\infty[30,38]$. This is done by making use of the conditions (2.8), (2.9), and (2.11) taken at $\Phi=0$. The condition (2.8) fixes the renormalization constant $\delta_{1}$; i.e.,

$$
\frac{\delta_{1}}{4 !}=\frac{\lambda^{2} \ln \left(\frac{\mu^{2}}{m^{2}}\right)}{256 \pi^{2} \sqrt{1+\chi}} .
$$

Furthermore, the renormalization conditions (2.9) and (2.11) fix, respectively, the constants $\delta_{2}$ and $\delta_{3}$, providing

$$
\frac{\delta_{2}}{2}=\frac{\lambda m^{2}}{64 \pi^{2} \sqrt{1+\chi}}+\frac{\lambda m^{2} \ln \left(\frac{\mu^{2}}{m^{2}}\right)}{64 \pi^{2} \sqrt{1+\chi}}
$$

and

$$
\delta_{3}=\frac{3 m^{4}}{128 \pi^{2} \sqrt{1+\chi}}+\frac{m^{4} \ln \left(\frac{\mu^{2}}{m^{2}}\right)}{64 \pi^{2} \sqrt{1+\chi}} .
$$

The renormalization constants above, when taken into account in Eq. (2.26), allow us to obtain the renormalized effective potential at one-loop level:

$$
\begin{aligned}
V_{\mathrm{eff}}^{\mathrm{R}}(\Phi)= & \frac{m^{2} \Phi^{2}}{2}+\frac{\lambda \Phi^{4}}{4 !}-\frac{3 b^{4}}{128 \pi^{2} \sqrt{1+\chi}}+\frac{3 m^{4}}{128 \pi^{2} \sqrt{1+\chi}}+\frac{b^{3}}{24 \pi \sqrt{1+\chi} a} \\
& +\frac{m^{2} \lambda \Phi^{2}}{64 \pi^{2} \sqrt{1+\chi}}+\frac{b^{4} \ln \left(\frac{b^{2}}{m^{2}}\right)}{64 \pi^{2} \sqrt{1+\chi}}-\frac{b^{2}}{8 \pi^{2} \sqrt{1+\chi} a^{2}} \sum_{n=1}^{\infty} \frac{K_{2}(2 b a n)}{n^{2}}
\end{aligned}
$$

This expression for the renormalized effective potential is clearly affected by the Lorentz symmetry violation parameter $\chi$, as it should be.

The renormalized effective potential, Eq. (2.30), when taken at the vacuum state $\Phi=0$, provides a nonvanishing vacuum Casimir-like potential energy by unit area of the plates, given by

$\frac{E_{C}}{L^{2}}=a V_{\mathrm{eff}}^{\mathrm{R}}(0)=-\frac{m^{2}}{8 \pi^{2} \sqrt{1+\chi} a} \sum_{n=1}^{\infty} \frac{K_{2}(2 a m n)}{n^{2}}$.

As we can see, the Casimir potential energy density above is affected by the Lorentz symmetry violation parameter $\chi$ through a multiplicative factor. Although this potential energy is given in terms of a sum of the modified Bessel functions, $K_{2}(2 \mathrm{man})$, it is a convergent expression, since for large values of $n$ this function is exponentially suppressed.

It is possible to provide closed expressions for the asymptotic behaviors, as $a m \gg 1$ and $a m \ll 1$, of the vacuum potential energy density. Thus, in the case $a m \gg 1$, by using the asymptotic expression for the modified Bessel function for large arguments [37], we get

$$
\frac{E_{C}}{L^{2}} \approx-\frac{1}{16 \sqrt{1+\chi}}\left(\frac{m}{\pi a}\right)^{3 / 2} e^{-2 a m}
$$

In this limit, the dominant term in Eq. (2.31) is for $n=1$, and we can clearly see that for large values of am the vacuum potential energy density is exponentially suppressed.

As to the case when am $\ll 1$, it is convenient first to use the integral representation for the modified Bessel function [39]:

$$
K_{\nu}(z)=\frac{\sqrt{\pi}\left(\frac{1}{2} z\right)^{\nu}}{\Gamma(\nu+1 / 2)} \int_{1}^{\infty} e^{-z t}\left(t^{2}-1\right)^{\nu-1 / 2} d t
$$

By substituting the above representation into Eq. (2.31), it is possible to develop the sum over $n$. Doing this, we get

$$
\frac{E_{C}}{L^{2}}=-\frac{a m^{4}}{6 \pi^{2} \sqrt{1+\chi}} \int_{1}^{\infty} d v \frac{\left(v^{2}-1\right)^{3 / 2}}{e^{2 a m v}-1} .
$$

In the regime of $a m \ll 1$, the integral in Eq. (2.34) is dominated by large values of $v$, so we may approximate Eq. (2.34) to 


$$
\frac{E_{C}}{L^{2}} \approx-\frac{a m^{4}}{6 \pi^{2} \sqrt{1+\chi}} \int_{1}^{\infty} d v \frac{v^{3}}{e^{2 a m v}-1}
$$

Now, we can obtain an expression to the integral in Eq. (2.35), which, developing a series expansion in powers of $a m \ll 1$, provides

$\frac{E_{C}}{L^{2}} \approx-\frac{\pi^{2}}{1440 \sqrt{1+\chi} a^{3}}+\frac{m^{3}}{36 \pi^{2} \sqrt{1+\chi}}-\frac{a m^{4}}{48 \pi^{2} \sqrt{1+\chi}}$.

Note that the leading term in the first term on the rhs of Eq. (2.36) is the contribution of the massless scalar field, which becomes an exact expression in the limit $m \rightarrow 0$. Moreover, we also recover from Eq. (2.31) results for the Casimir effect for a real scalar field which satisfies Dirichlet and Neumann boundary conditions on two parallel plates without Lorentz violation $[36,40,41]$.

As has been said before, the two-loop contribution to the effective potential comes from the two graphs to the effective Euclidian action given in Refs. [30,31]. As we are only interested in the two-loop contribution to the vacuum energy, its only nonzero term, at $\Phi=0$, is given by

$$
V^{(2)}(\Phi=0)=\frac{\lambda}{8} S_{1}(\Phi=0)
$$

The function $S_{1}(\Phi)$ is obtained by means of the expression

$$
\begin{aligned}
S_{1}(\Phi)= & \left\{\sum _ { n = 1 } ^ { \infty } \frac { 1 } { a } \int \frac { d ^ { 3 } k } { ( 2 \pi ) ^ { 3 } } \left[(1+\chi) k_{t}^{2}+k^{2}\right.\right. \\
& \left.\left.+\frac{\pi^{2} n^{2}}{a^{2}}+m^{2}+\frac{\lambda \Phi^{2}}{2}\right]^{-s}\right\}^{2},
\end{aligned}
$$

where $s$ has been introduced in order to regularize the expression above. After we subtract the divergent part of Eq. (2.38), we should take $s=1$. This allows us to write the finite contribution of the function $S_{1}(\Phi)$ in terms of the zeta function (2.21) as

$$
S_{1}(\Phi)=\left[\frac{\zeta_{R}(1)}{V_{3} a}\right]^{2}
$$

Note that $\zeta_{R}(1)$ is the zeta function [Eq. (2.21)] taken at $s=1$ after subtracting the divergent part of it given by the second term on the rhs. This term, when divided by $V_{3} a$, is independent of $a$ and presents a divergent contribution at $s=1$ proportional to $\frac{\Gamma(s-2)}{\Gamma(s)} \approx \frac{s}{1-s}$. As is usually done, this term should be subtracted, since it does not depend on the boundary condition parameter - that is, $a$. Hence, Eqs. (2.37) and (2.39) provide the two-loop contribution to the effective potential as
$\frac{E_{C}^{(\lambda)}}{L^{2}}=a V^{(2)}(0)=\frac{m^{2} \lambda}{128 \pi^{4}(1+\chi) a}\left[\sum_{n=1}^{\infty} \frac{K_{1}(2 a m n)}{n}\right]^{2}$

Also, we can see that $\frac{E_{C}^{(\lambda)}}{L^{2}}$ depends on the Lorentz violation parameter by a multiplicative factor; moreover, it is an exact and a convergent function, which can be seen by noting that the modified Bessel function $K_{1}(2 \mathrm{man})$ is exponentially suppressed for large values of $n$.

We can also obtain closed expressions for Eq. (2.40) in the regimes where $a m \gg 1$ and $a m \ll 1$. Considering first the case $a m \gg 1$, we have

$$
\frac{E_{C}^{(\lambda)}}{L^{2}} \approx \frac{\lambda m e^{-4 a m}}{512 \pi^{3}(1+\chi) a^{2}}
$$

which is dominated by the term $n=1$ in the sum and is exponentially suppressed. This feature is shown in Fig. 2.

For the opposite regime-that is, when am $\ll 1$-we should use again the integral representation in Eq. (2.33) for the modified Bessel function $K_{\mu}(x)$. Developing a similar procedure as before, we obtain

$$
\frac{E_{C}^{(\lambda)}}{L^{2}} \approx \frac{\lambda}{18432(1+\chi) a^{3}}-\frac{\lambda m}{768 \pi^{2}(1+\chi) a^{2}},
$$

which give us as the leading contribution the massless scalar field expression in the first term on the rhs of it. This becomes an exact expression in the limit $m \rightarrow 0$.

In the left panel of Fig. 2, we exhibit the behavior of the Casimir energy per unit area given by Eq. (2.31), as a function of the dimensionless parameter $m a$, considering different values for the parameter $\chi$. We can see that it increases as the Lorentz symmetry violation parameter increases. In the right plot, on the other hand, we exhibit the two-loop correction to the Casimir energy per unit area, Eq. (2.40), as a function of $\mathrm{am}$. It is clear that both plots are in agreement with the asymptotic behaviors (2.32) and (2.36) for the Casimir energy, and with (2.41) and (2.42) for the two-loop correction.

At one-loop level, the massive scalar field we are considering will get quantum corrections to its mass. This correction can be obtained by using condition (2.9), at $\Phi=0$, for the renormalized effective potential [Eq. (2.30)] at one-loop level; i.e.,

$$
\begin{aligned}
m_{\mathrm{T}}^{2}= & \left.\frac{d^{2} V_{\mathrm{eff}}^{\mathrm{R}}(\Phi)}{d \Phi^{2}}\right|_{\Phi=0} \\
= & m^{2}\left[1+\frac{\lambda}{16 \pi \sqrt{1+\chi} a m}\right. \\
& \left.+\frac{\lambda}{8 \pi^{2} \sqrt{1+\chi} a m} \sum_{n=1}^{\infty} \frac{K_{1}(2 a m n)}{n}\right] .
\end{aligned}
$$



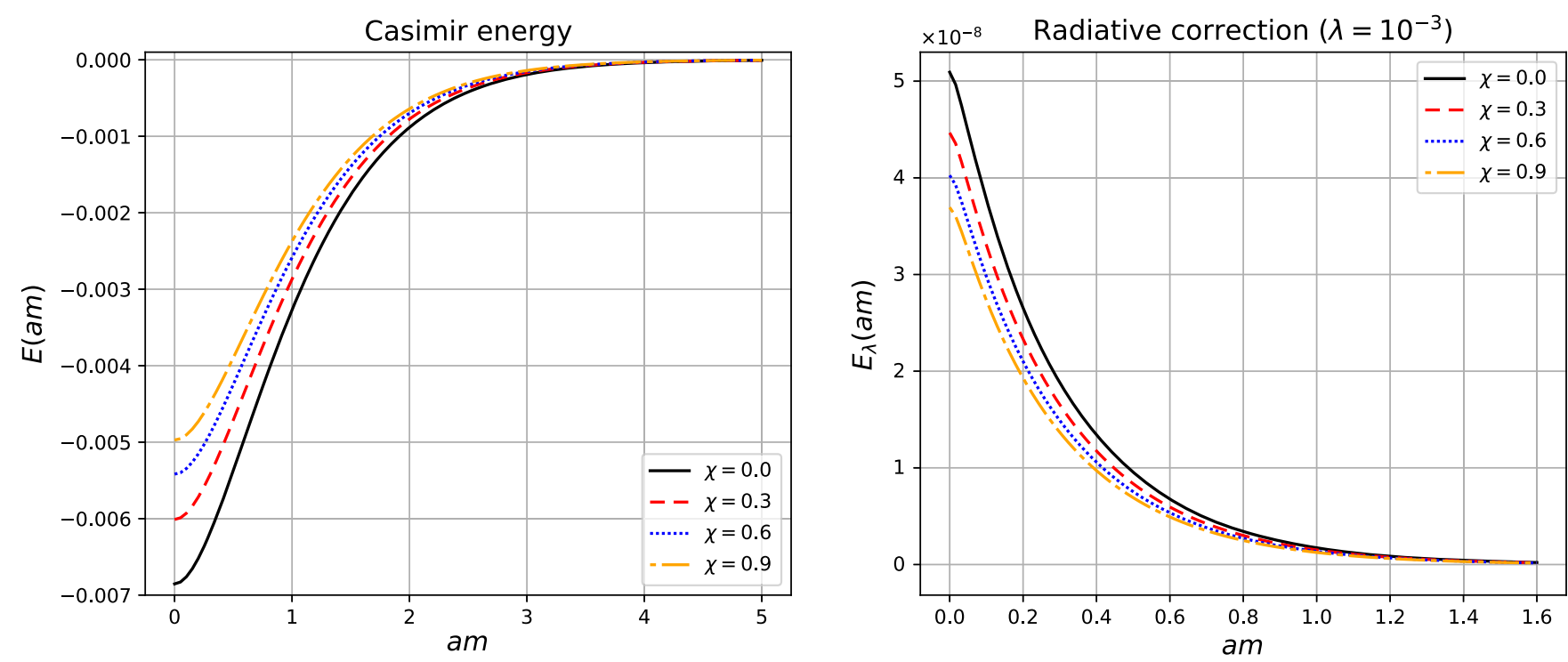

FIG. 2. The behavior of the Casimir energy per unit area $E(a m)=\frac{a^{3}}{L^{2}} E_{C}$ given by Eq. (2.31) as a function of $a m$ is exhibited in the left panel, and the two-loop contribution to the Casimir energy per unit area $E_{\lambda}(a m)=\frac{a^{3}}{L^{2}} E_{C}^{(\lambda)}$ in Eq. (2.40) as a function of $a m$ is presented in the right panel, considering $\lambda=10^{-3}$. For both plots, different values for the parameter $\chi$ are considered.

This expression presents a topological contribution, which depends on $a$, to the mass $m$ of the scalar field given by the terms proportional to the self-coupling constant $\lambda$. Even for the massless scalar field, which remains massless at the tree level, there appears a topological mass generated at the oneloop correction, as we can see from Eq. (2.43). Moreover, the third term inside the bracket is convergent, since it is exponentially suppressed for large values of $n$. In fact, the leading contribution for $a m \gg 1$ is given by the first term on the rhs of Eq. (2.43). This asymptotic behavior can be obtained by using the expressions for the modified Bessel function for large arguments [37]. The asymptotic expression for Eq. (2.43) in the regime $a m \gg 1$ is

$m_{\mathrm{T}}^{2} \approx m^{2}+\frac{\lambda m}{16 \pi \sqrt{1+\chi} a}+\frac{\lambda}{16 \pi^{3 / 2}} \sqrt{\frac{m}{a^{3}}} \frac{e^{-2 a m}}{\sqrt{1+\chi}}$.

On the other hand, for $a m \ll 1$, the leading term is mass independent, followed by terms that depend on the mass of the scalar field. Once more, this behavior can be obtained by using the integral representation for the function $K_{\mu}(z)$, Eq. (2.33). After some intermediate steps, we obtain

$m_{\mathrm{T}}^{2} \approx \frac{\lambda}{96 \sqrt{1+\chi} a^{2}}+\frac{\lambda m}{16 \pi \sqrt{1+\chi} a}-\frac{\lambda m}{8 \pi^{2} \sqrt{1+\chi} a}+m^{2}$.

The asymptotic results, Eqs. (2.44) and (2.45), are in agreement with the plot of Fig. 3, which exhibits the behavior of the ratio of the topological mass to the field's mass itself, $m_{T} / m$, as a function of am for different parameters $\chi$. Note that the topological mass decreases as $\chi$ increases.

\section{Spacelike vector}

Now, we consider the case that the constant fourvector is spacelike - that is, of the types $u^{x}=(0,1,0,0)$, $u^{y}=(0,0,1,0)$, or $u^{z}=(0,0,0,1)$. The first two types are parallel to the plates and provide essentially the same results, while the third type is perpendicular to the plates. Let us then begin by considering the parallel cases by assuming, for instance, that a breaking of Lorentz symmetry happens in the $x$ direction-i.e., $u^{x}=(0,1,0,0)$. This gives us the set of eigenvalues

$$
\Lambda_{\beta}=k^{2}+(1-\chi) k_{x}^{2}+\frac{\pi^{2} n^{2}}{a^{2}}+m^{2}+\frac{\lambda}{2} \Phi^{2},
$$

where $k^{2}=k_{t}^{2}+k_{y}^{2}$. Thereby, from Eq. (2.7), the set of eigenvalues above is associated with the elliptic operator given by

$$
\Delta=-\partial_{\mu} \partial^{\mu}+\chi \partial_{x}^{2}+m^{2}+\frac{\lambda}{2} \Phi^{2} .
$$

So, in this case, the zeta function (2.6) is written as

$$
\begin{aligned}
\zeta(s)= & \frac{V_{3}}{(2 \pi)^{3}} \int d^{3} k \\
& \times \sum_{n=1}^{\infty}\left[k^{2}+(1-\chi) k_{x}^{2}+\frac{\pi^{2} n^{2}}{a^{2}}+m^{2}+\frac{\lambda}{2} \Phi^{2}\right]^{-s} .
\end{aligned}
$$




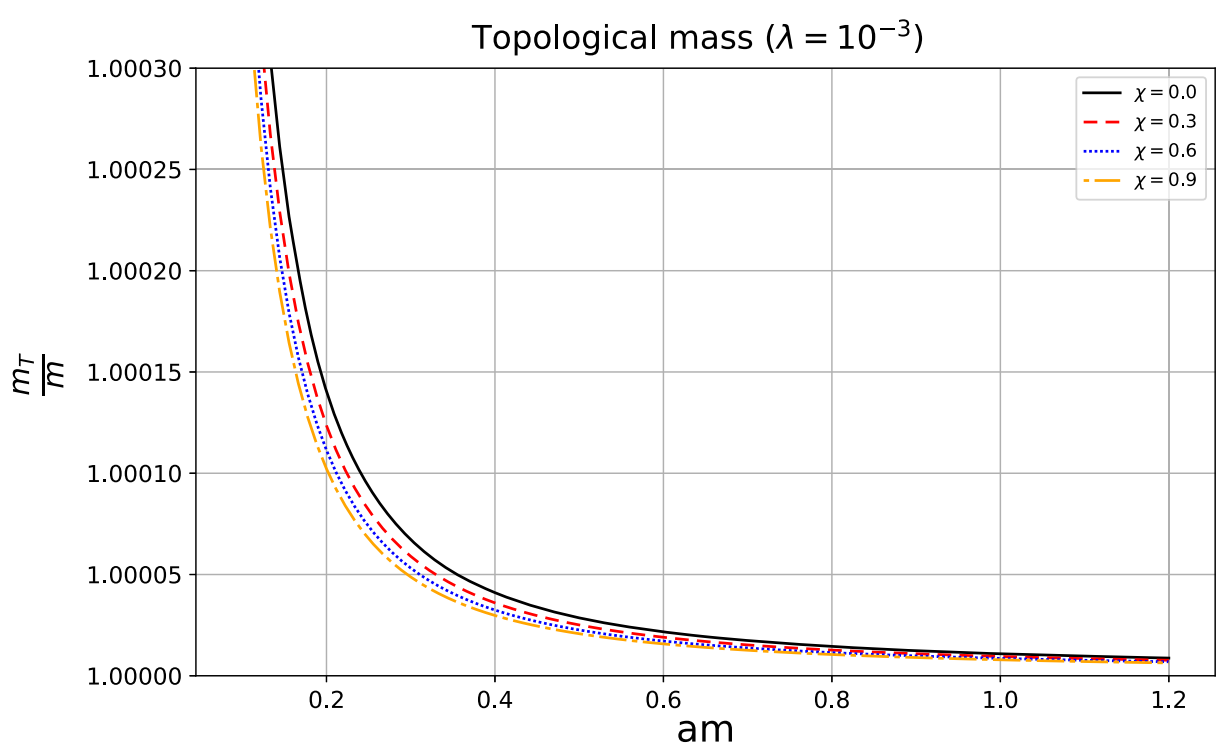

FIG. 3. The behavior of the ratio of the topological mass to the scalar field mass as a function of $a m$ is plotted assuming $\lambda=10^{-3}$ and different values for the Lorentz-violating parameter $\chi$.

The integrals in $k_{t}, k_{x}$, and $k_{y}$ can be solved using the identity (2.16), providing

$\zeta(s)=\frac{V_{3}}{(2 \pi)^{3}} \frac{\pi^{3 / 2}}{\sqrt{1-\chi}} \frac{\Gamma(s-3 / 2)}{\Gamma(s)} w^{3-2 s} \sum_{n=1}^{\infty}\left(n^{2}+\nu^{2}\right)^{3 / 2-s}$,

where

$$
\nu^{2}=\frac{\lambda \Phi^{2}}{2 w^{2}}+\frac{m^{2}}{w^{2}} \quad \text { and } \quad w=\frac{\pi}{a}
$$

Clearly, we can see that this result is very similar to the one obtained in the timelike case, with a different dependence on the Lorentz violation parameter, $\chi$. In fact, the timelike and spacelike expressions for the zeta function, Eqs. (2.17) and (2.49), respectively, are related by the change $\chi \rightarrow-\chi$. Hence, we expect that all the results for the vacuum energy, its loop correction, and the topological mass will be related by this same change.

Let us now turn to the most important type of spacelike Lorentz symmetry violation-namely, the one in the perpendicular direction to the plates given by

$$
u^{z}=(0,0,0,1)
$$

This provides, from Eq. (2.7), the following differential elliptic operator:

$$
\Delta=-\partial_{\mu} \partial^{\mu}+\chi \partial_{z}^{2}+m^{2}+\frac{\lambda \Phi^{2}}{2} .
$$

Consequently, the set of eigenvalues associated with this operator is found to be

$$
\Lambda_{\beta}=k^{2}+(1-\chi) \frac{\pi^{2} n^{2}}{a^{2}}+m^{2}+\frac{\lambda \Phi^{2}}{2} .
$$

Note that $k^{2}=k_{t}^{2}+k_{x}^{2}+k_{y}^{2}$. Thereby, the zeta function (2.6), taking into consideration Eq. (2.53), is written as

$$
\zeta(s)=\frac{V_{3}}{(2 \pi)^{3}} \sum_{n=1}^{\infty} \int d^{3} k\left[k^{2}+(1-\chi) \frac{\pi^{2} n^{2}}{a^{2}}+m^{2}+\frac{\lambda \Phi^{2}}{2}\right]^{-s},
$$

where, again, $V_{3}$ is the continuum volume associated with the dimensions $t, x, y$ and $d^{3} k=d k_{t} d k_{x} d k_{y}$. Thus, the identity (2.16) allows us to perform the integrals in $k_{t}, k_{x}$, and $k_{y}$ in Eq. (2.54). The resulting expression, in analogy with Eq. (2.49), can be written in terms of the EpsteinHurwitz function (2.19), providing that

$$
\begin{aligned}
\zeta(s)= & \frac{V_{3}}{(2 \pi)^{3}}\left[-\frac{\pi^{3 / 2} w^{-2 \bar{s}} \nu^{-2 \bar{s}}}{2} \frac{\Gamma(\bar{s})}{\Gamma(\bar{s}+3 / 2)}+\frac{\pi^{1 / 2} \pi^{3 / 2} w^{-2 \bar{s}}}{2} \frac{\Gamma(\bar{s}-1 / 2)}{\Gamma(\bar{s}+3 / 2)} \nu^{1-2 \bar{s}}\right. \\
& \left.+\frac{2^{1-\bar{s}}(2 \pi)^{2 \bar{s}-1 / 2} \pi^{3 / 2} w^{-2 \bar{s}}}{\Gamma(\bar{s}+3 / 2)} \sum_{n=1}^{\infty} n^{2 \bar{s}-1} f_{(\bar{s}-1 / 2)}(2 \pi n \nu)\right],
\end{aligned}
$$


where $\bar{s}=s-3 / 2$, and

$$
\nu^{2}=\frac{\lambda \Phi^{2}}{2 w^{2}}+\frac{m^{2}}{w^{2}} \quad \text { and } \quad w \equiv \sqrt{1-\chi} \frac{\pi}{a} .
$$

Note that one should consider the limit $s \rightarrow 0$, or analogously $\bar{s} \rightarrow-3 / 2$. In this limit, we can get the expressions for $\zeta(0)$ and $\zeta^{\prime}(0)$, respectively, given by

$$
\begin{gathered}
\zeta(0)=\frac{V_{3} a}{2(2 \pi)^{4}} \frac{\pi^{2} b^{4}}{\sqrt{1-\chi}}, \\
\zeta^{\prime}(0)=\frac{V_{3} L}{(2 \pi)^{3}}\left[-\frac{2 \pi^{2}}{3} \frac{b^{3}}{a}+\frac{3}{8} \frac{\pi b^{4}}{\sqrt{1-\chi}}-\frac{\pi}{2} \frac{b^{4} \ln (b)}{\sqrt{1-\chi}}\right. \\
\left.+\frac{2 \pi \sqrt{1-\chi} b^{2}}{a^{2}} \sum_{n=1}^{\infty} \frac{K_{2}\left(\frac{2 a b n}{\sqrt{1-\chi}}\right)}{n^{2}}\right] .
\end{gathered}
$$

Consequently, from Eq. (2.5), the one-loop correction to the effective potential is

$$
\begin{aligned}
V^{(1)}(\Phi)= & \frac{b^{3}}{24 \pi a}-\frac{3 b^{4}}{128 \pi^{2} \sqrt{1-\chi}}+\frac{b^{4} \ln \left(\frac{b^{2}}{\mu^{2}}\right)}{64 \pi^{2} \sqrt{1-\chi}} \\
& -\frac{b^{2} \sqrt{1-\chi}}{8 \pi^{2} a^{2}} \sum_{n=1}^{\infty} \frac{K_{2}\left(\frac{2 a b n}{\sqrt{1-\chi}}\right)}{n^{2}},
\end{aligned}
$$

where the parameter $b$ is defined as

$$
b=\sqrt{\frac{\lambda \Phi^{2}}{2}+m^{2}} .
$$

Hence, the effective potential up to one-loop correction, given by the expression (2.4), is obtained as

$$
\begin{aligned}
V_{\text {eff }}(\Phi)= & \frac{m^{2} \Phi^{2}}{2}+\frac{\lambda \Phi^{4}}{4 !}+\frac{\Phi^{2}}{2} \delta_{2}+\frac{\Phi^{4}}{4 !} \delta_{1}+\delta_{3} \\
& +\frac{b^{3}}{24 \pi a}-\frac{3 b^{4}}{128 \pi^{2} \sqrt{1-\chi}} \\
& +\frac{b^{4} \ln \left(\frac{b^{2}}{\mu^{2}}\right)}{64 \pi^{2} \sqrt{1-\chi}}-\frac{b^{2} \sqrt{1-\chi}}{8 \pi^{2} a^{2}} \sum_{n=1}^{\infty} \frac{K_{2}\left(\frac{2 a b n}{\sqrt{1-\chi}}\right)}{n^{2}} .
\end{aligned}
$$

We should now obtain the renormalization constants $\delta_{1}, \delta_{2}$, and $\delta_{3}$, which can be done by using the conditions (2.8), (2.9), and (2.11), respectively. Thus, the renormalization constant $\delta_{1}$ is found to be

$$
\begin{gathered}
\frac{\delta_{1}}{4 !}=\frac{\lambda^{2} \ln \left(\frac{\mu^{2}}{m^{2}}\right)}{256 \pi^{2} \sqrt{1-\chi}}, \\
\frac{\delta_{2}}{2 !}=\frac{m^{2} \lambda}{64 \pi^{2} \sqrt{1-\chi}}+\frac{m^{2} \lambda \ln \left(\frac{\mu^{2}}{m^{2}}\right)}{64 \pi^{2} \sqrt{1-\chi}},
\end{gathered}
$$

and

$$
\delta_{3}=\frac{3 m^{4}}{128 \pi^{2} \sqrt{1-\chi}}+\frac{m^{4} \ln \left(\frac{\mu^{2}}{m^{2}}\right)}{64 \pi^{2} \sqrt{1-\chi}} .
$$

Hence, substituting the renormalization constants above in Eq. (2.61), the renormalized effective potential, up to oneloop correction, is found to be

$$
\begin{aligned}
V_{\mathrm{eff}}^{\mathrm{R}}(\Phi)= & \frac{m^{2} \Phi^{2}}{2}+\frac{\lambda \Phi^{4}}{4 !}+\frac{b^{3}}{24 \pi a}-\frac{3 b^{4}}{128 \pi^{2} \sqrt{1-\chi}}+\frac{m^{2} \lambda \Phi^{2}}{64 \pi^{2} \sqrt{1-\chi}}+\frac{3 m^{4}}{128 \pi^{2} \sqrt{1-\chi}} \\
& +\frac{b^{4}}{64 \pi^{2} \sqrt{1-\chi}} \ln \left(\frac{b^{2}}{m^{2}}\right)-\frac{b^{2} \sqrt{1-\chi}}{8 \pi^{2} a^{2}} \sum_{n=1}^{\infty} \frac{K_{2}\left(\frac{2 a b n}{\sqrt{1-\chi}}\right)}{n^{2}} .
\end{aligned}
$$

The vacuum energy per unit area of the plates is obtained when we take the vacuum state $(\Phi=0)$. Thus, from Eq. (2.65), we get

$$
\frac{E_{\mathrm{C}}}{L^{2}}=a V_{\mathrm{eff}}^{R}(0)=-\frac{m^{2} \sqrt{1-\chi}}{8 \pi^{2} a} \sum_{n=1}^{\infty} \frac{K_{2}\left(\frac{2 a m n}{\sqrt{1-\chi}}\right)}{n^{2}},
$$

which is convergent, and therefore finite. This expression for the vacuum energy per unit area is exponentially suppressed for $m a \gg 1$ and provides the massless scalar field expression for $m a \ll 1$.

We can mathematically obtain an asymptotic expression for Eq. (2.66) in the regimes $m a \ll 1$ and $m a \gg 1$. By considering the latter, we have

$$
\frac{E_{C}}{L^{2}} \approx-\frac{\sqrt{1-\chi}}{16}\left(\frac{m}{\pi a}\right)^{3 / 2} e^{-\frac{2 a m}{\sqrt{1-\chi}}}
$$

which, as mentioned before, is exponentially suppressed. It is the dominant term for $n=1$ in the sum.

As to the limit $a m \ll 1$, we should first use the integral representation for the modified Bessel function, $K_{\mu}(x)$, in Eq. (2.33). This provides

$$
\frac{E_{C}}{L^{2}} \approx-\frac{\pi^{2}(1-\chi)^{3 / 2}}{1440 a^{3}}+\frac{m^{3}}{36 \pi^{2}}-\frac{a m^{4}}{48 \pi^{2} \sqrt{1-\chi}} .
$$



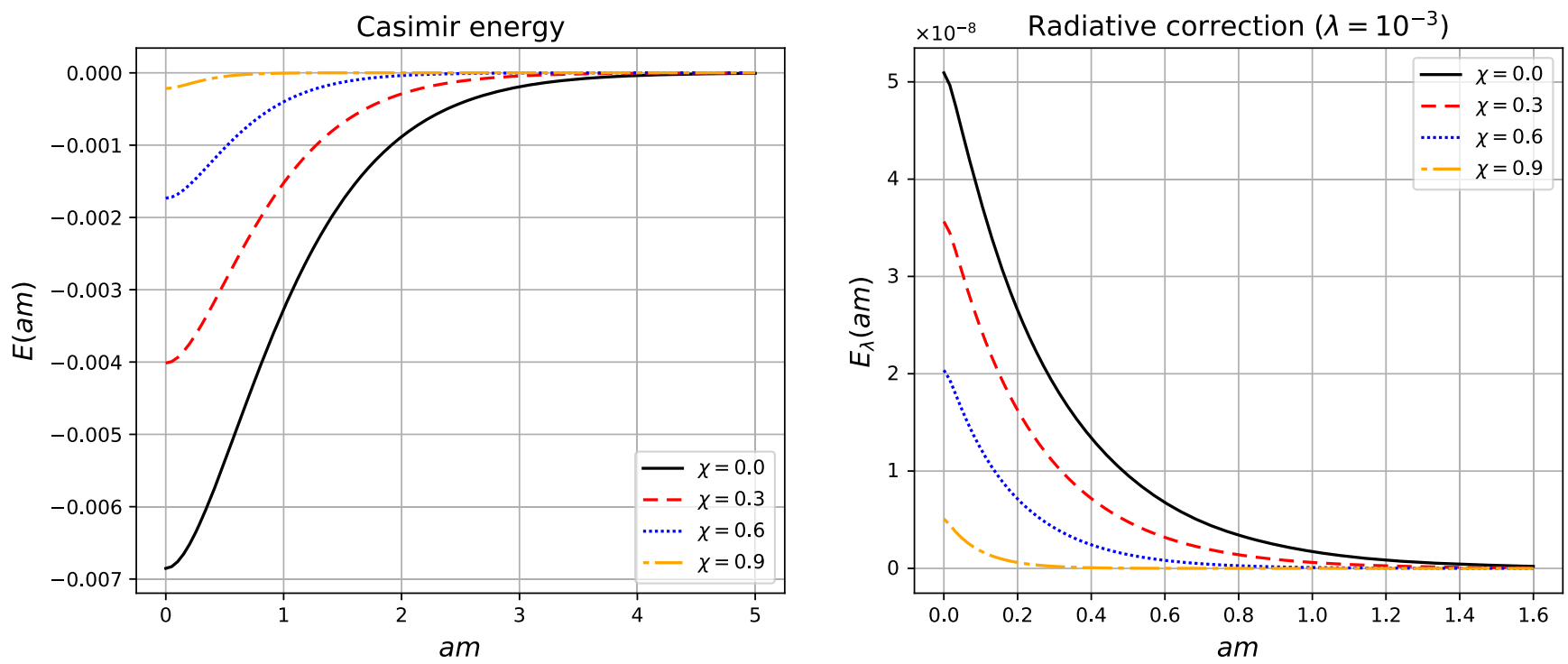

FIG. 4. The behavior of the Casimir energy per unit area $E(a m)=\frac{a^{3}}{L^{2}} E_{C}$ given by Eq. (2.66) as a function of $a m$ is presented in the left panel, and the two-loop correction $E_{\lambda}(a m)=\frac{a^{3}}{L^{2}} E_{C}^{(\lambda)}$ given by Eq. (2.70) as a function of $a m$ is in the right one. In the latter, we have taken $\lambda=10^{-3}$ and considered different values for $\chi$.

Note that the dominant term is the first one on the rhs and represents the vacuum energy per unit area in the massless scalar field case.

Let us turn to the two-loop contribution to the effective potential calculated at $\Phi=0$ in the case in which the fourvector is orthogonal to the parallel plates. The $S_{1}(\Phi)$ function in Eq. (2.37) is now written as

$$
\begin{aligned}
S_{1}(\Phi)= & \left\{\sum_{n=1}^{\infty} \frac{1}{a} \int \frac{d^{3} k}{(2 \pi)^{3}}\right. \\
& \left.\times\left[k^{2}+(1-\chi) \frac{\pi^{2} n^{2}}{a^{2}}+m^{2}+\frac{\lambda \Phi^{2}}{2}\right]^{-s}\right\}^{2},
\end{aligned}
$$

which should be taken at $s=1$ after subtracting the divergent contribution. This can be done by using the zeta function (2.55) in a similar way as in the previous case shown in Eq. (2.39). In the present case, the divergent contribution comes from the second term on the rhs of Eq. (2.55) and, after subtracted, the two-loop contribution $V^{(2)}(0)$ at $s=1$ can be found, leading to the two-loop correction to the vacuum energy:

$$
\frac{E_{C}^{(\lambda)}}{L^{2}}=a V^{(2)}(0)=\frac{m^{2} \lambda}{128 \pi^{4} a}\left[\sum_{n=1}^{\infty} \frac{K_{1}\left(\frac{2 a m n}{\sqrt{1-\chi}}\right)}{n}\right]^{2} .
$$

This is a completely convergent expression, since the modified Bessel function, $K_{\mu}(x)$, is exponentially suppressed. This is also clear when one considers the asymptotic limit $m a \gg 1$. In the opposite limit, $m a \ll 1$, the expression for the two-loop contribution to the vacuum energy for a massless scalar field is obtained, as the leading contribution.
We can also obtain the asymptotic expressions for Eq. (2.70) in the regimes $m a \ll 1$ and $m a \gg 1$. In the latter, we have

$$
\frac{E_{C}^{(\lambda)}}{L^{2}} \approx \frac{\lambda m \sqrt{1-\chi} e^{-\frac{4 a m}{\sqrt{1-\chi}}}}{512 \pi^{3} a^{2}},
$$

which is exponentially suppressed and dominated by the term $n=1$ in the sum.

In the opposite limit, $m a \ll 1$, we need to use the integral representation (2.33) for the modified Bessel function, $K_{\mu}(x)$. This provides

$$
\frac{E_{C}^{(\lambda)}}{L^{2}} \approx \frac{\lambda(1-\chi)}{18432 a^{3}}-\frac{\lambda m \sqrt{1-\chi}}{768 \pi^{2} a^{2}}
$$

Note that the first term on the rhs is the massless scalar field contribution which becomes exact when $m a \rightarrow 0$.

In the left panel of Fig. 4, we plot the behavior of the Casimir energy per unit area [Eq. (2.66)] as a function of am considering different values for the parameter $\chi$. This plot shows that as $\chi$ increases, the vacuum energy also increases. On the other hand, in the right figure we plot the two-loop correction to the vacuum energy per unit area [Eq. (2.70)] as a function of $a m$, assuming $\lambda=10^{-3}$. This plot shows that as $\chi$ increases, the two-loop correction decreases. Moreover, one should note an important feature here-namely, the vacuum energy (2.66) and its radiative correction (2.70) not only depend on $\chi$ by means of a multiplicative factor but also depend on $\chi$ in the argument of the modified Bessel function, $K_{\mu}(x)$. This stronger 


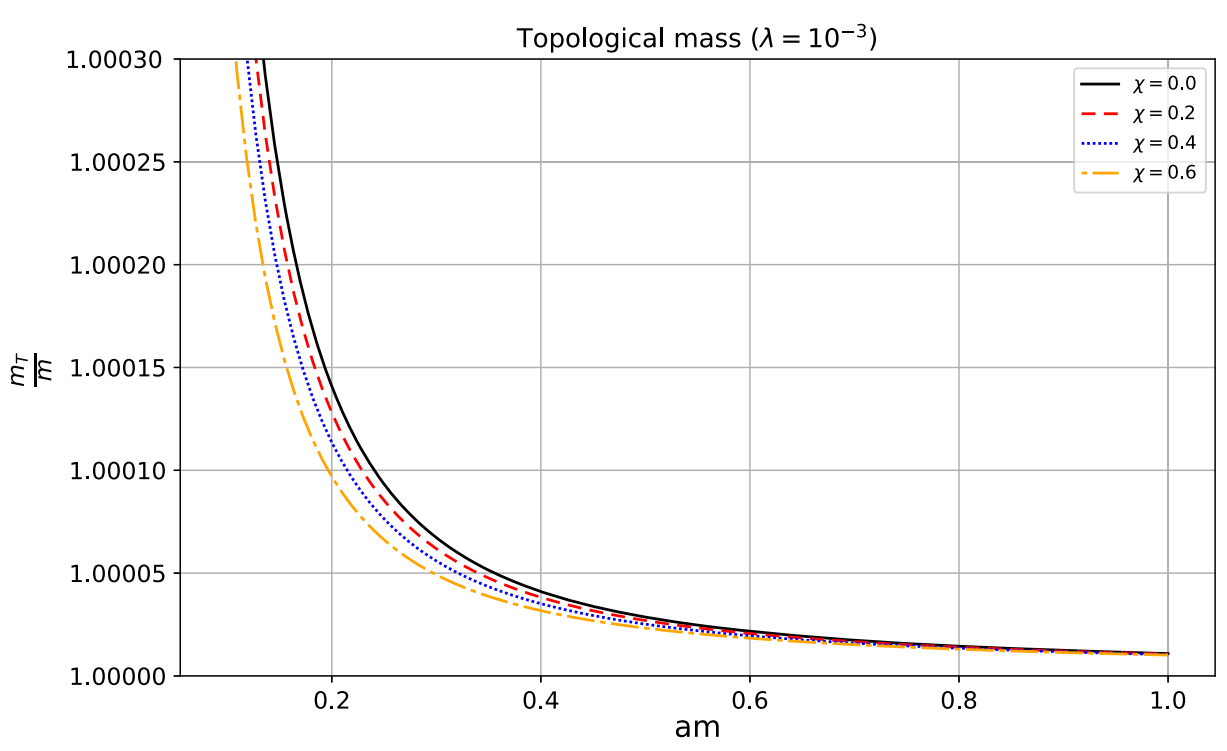

FIG. 5. Plot exhibiting the behavior of the ratio of the topological mass by the mass of the field as a function of $a m$. The plot considers $\lambda=10^{-3}$ and different variations of $\chi$.

dependence is shown in the plots of Fig. 4. The shift in the curves is stronger than in the previous timelike case.

A topological mass in this case will also be generated and can be obtained by using the condition (2.9). Thus, by applying the latter in the renormalized effective potential [Eq. (2.65)], we find

$m_{\mathrm{T}}^{2}=m^{2}\left[1+\frac{\lambda}{16 \pi a m}+\frac{\lambda}{8 \pi^{2} a m} \sum_{n=1}^{\infty} \frac{K_{1}\left(\frac{2 a m n}{\sqrt{1-\chi}}\right)}{n}\right]$.

This is an exact convergent expression, and in the limit $m a \gg 1$ is dominated by the first term on the rhs, while in the limit $m a \ll 1$ is dominated by the third term, the massless scalar field contribution.

Mathematically, the asymptotic behavior $m a \gg 1$ of Eq. (2.73) is given by

$$
m_{\mathrm{T}}^{2} \approx m^{2}+\frac{\lambda m}{16 \pi a}+\frac{\lambda \sqrt[4]{1-\chi}}{16 \pi^{3 / 2}} \sqrt{\frac{m}{a^{3}}} e^{-\frac{2 a m}{\sqrt{1-\chi}}}
$$

while the asymptotic behavior $m a \ll 1$ is

$$
m_{\mathrm{T}}^{2} \approx \frac{\lambda \sqrt{1-\chi}}{96 a^{2}}+\frac{\lambda m}{16 \pi a}+m^{2}
$$

The asymptotic results, Eqs. (2.74) and (2.75), are in agreement with the plot of Fig. 5, which exhibits the behavior of the ratio of the topological mass to the field's mass as a function of $m a$ for a fixed value of $\lambda$ and different values of $\chi$. Note that, due to the dependence of the topological mass (2.73) on $\chi$ in the argument of the modified Bessel function, $K_{\mu}(x)$, the curves in Fig. 5 are shifted down as $\chi$ increases, more than in the timelike case.
It is important to point out that all the results obtained up to this point, adopting the Dirichlet boundary condition for the timelike and spacelike types of Lorentz symmetry violation, are the same ones obtained when we consider the Neumann boundary condition, as it should be. In the next section, we will consider a mix of these two boundary conditions, which we will refer to as a mixed boundary condition.

\section{Mixed boundary condition}

After the analysis of the effective potential, Casimir-like effect, and topological mass assuming Dirichlet and Neumann boundary conditions obeyed by a massive scalar field on two parallel plates, now we want to consider the case in which the field satisfies a mixed boundary condition. In other words, we assume that the field obeys Dirichlet and Neumann boundary conditions on each of the plates separately. The conditions are then written as

$$
\left.\varphi(x)\right|_{z=0}=\left.\frac{\partial \varphi(x)}{\partial z}\right|_{z=a}=0 \quad \text { and }\left.\quad \frac{\partial \varphi(x)}{\partial z}\right|_{z=0}=\left.\varphi(x)\right|_{z=a} .
$$

The complete set of normalized solutions of the scalar field, $\varphi$, under these conditions has also been reported in Ref. [36]. These solutions provide the following eigenvalues of the operator (2.7):

$\Lambda_{\beta}=k_{t}^{2}+k^{2}+\left[\left(n+\frac{1}{2}\right) \frac{\pi}{a}\right]^{2}-\chi u^{\mu} u^{\nu} k_{\mu} k_{\nu}+m^{2}+\frac{\lambda \Phi^{2}}{2}$,

where $k^{2}=k_{x}^{2}+k_{y}^{2}$ and $n=0,1,2,3, \ldots$ Hence, we will consider the set of eigenvalues (2.77) representing 
the mixed boundary condition case to calculate the renormalized effective potential, the vacuum energy, and topological mass, taking into consideration the two types of Lorentz symmetry violation-namely, the timelike and spacelike types.

\section{Timelike vector}

In the case that the four-vector $u^{\mu}$ is of the timelike type-i.e., $u^{t}=(1,0,0,0)$ - the eigenvalues (2.77) of the elliptic operator (2.7) become

$$
\Lambda_{\beta}=(1+\chi) k_{t}^{2}+k^{2}+\left[\left(n+\frac{1}{2}\right) \frac{\pi}{a}\right]^{2}+m^{2}+\frac{\lambda \Phi^{2}}{2} .
$$

Consequently, the zeta function (2.6) is now written as

$$
\zeta(s)=\frac{1}{\sqrt{1+\chi}} \frac{V_{3}}{(2 \pi)^{3}} \sum_{n=0}^{\infty} \int d^{3} k\left\{\kappa_{t}^{2}+k^{2}+\left[\left(n+\frac{1}{2}\right) \frac{\pi}{a}\right]^{2}+m^{2}+\frac{\lambda \Phi^{2}}{2}\right\}^{-s} .
$$

Furthermore, by using the identity (2.16), we are able to perform the integrals in $\kappa_{t}, k_{x}$, and $k_{y}$, providing that

$$
\zeta(s)=\frac{1}{\sqrt{1+\chi}} \frac{V_{3}}{(2 \pi)^{3}} \frac{\pi^{3 / 2} \Gamma(s-3 / 2)}{\Gamma(s)} w^{3-2 s} \sum_{n=0}^{\infty}\left[\left(n+\frac{1}{2}\right)^{2}+\nu^{2}\right]^{3 / 2-s},
$$

where

$$
\nu^{2}=\frac{\lambda \Phi^{2}}{2 w^{2}}+\frac{m^{2}}{w^{2}} \quad \text { and } \quad w=\frac{\pi}{a} .
$$

We can see that there is still a sum in $n$ to be performed in the zeta function expression [Eq. (2.80)]. In this sense, in order to apply the Epstein-Hurwitz zeta function (2.19), we can write the sum in $n$ as

$$
\sum_{n=0}^{\infty}\left[\left(n+\frac{1}{2}\right)^{2}+\nu^{2}\right]^{3 / 2-s}=\frac{1}{2^{3-2 s}}\left[\sum_{n=1}^{\infty}\left(n^{2}+4 \nu^{2}\right)^{3 / 2-s}-2^{3-2 s} \sum_{n=1}^{\infty}\left(n^{2}+\nu^{2}\right)^{3 / 2-s}\right] .
$$

By using now Eqs. (2.82) and (2.19) in Eq. (2.80), we have the final form of the generalized zeta function as given by

$$
\begin{aligned}
\zeta(s)= & \frac{V_{3}}{(2 \pi)^{3}}\left\{\frac{\pi^{2} w^{-2 \bar{s}} \nu^{1-2 \bar{s}} \Gamma(\bar{s}-1 / 2)}{2 \Gamma(\bar{s}+3 / 2) \sqrt{1+\chi}}+\frac{2^{\bar{s}+1 / 2} \pi^{2 \bar{s}+1} w^{-2 \bar{s}}}{\Gamma(\bar{s}+3 / 2) \sqrt{1+\chi}}\right. \\
& \left.\times\left[4^{\bar{s}} \sum_{n=1}^{\infty} n^{2 \bar{s}-1} f_{\bar{s}-1 / 2}(4 \pi n \nu)-\sum_{n=1}^{\infty} n^{2 \bar{s}-1} f_{\bar{s}-1 / 2}(2 \pi n \nu)\right]\right\},
\end{aligned}
$$

where $\bar{s}=s-3 / 2$. Thus, by using Eq. (2.83), we can obtain $\zeta(0)$ and $\zeta^{\prime}(0)$, leading to the one-loop correction to the effective potential (2.5), written as

$$
\begin{aligned}
V^{(1)}(\Phi)= & -\frac{3 b^{4}}{128 \pi^{2} \sqrt{1+\chi}}+\frac{b^{4} \ln \left(\frac{b^{2}}{\mu^{2}}\right)}{64 \pi^{2} \sqrt{1+\chi}}-\frac{b^{2}}{16 \pi^{2} \sqrt{1+\chi} a^{2}} \\
& \times \sum_{n=1}^{\infty} \frac{\left[K_{2}(4 b a n)-2 K_{2}(2 b a n)\right]}{n^{2}}
\end{aligned}
$$

where

$$
b=\sqrt{\frac{\lambda \Phi^{2}}{2}+m^{2}} .
$$

Hence, from Eqs. (2.3) and (2.84), the effective potential up to one-loop correction is found to be 


$$
\begin{aligned}
V_{\mathrm{eff}}(\Phi)= & \frac{m^{2} \Phi^{2}}{2}+\frac{\lambda \Phi^{4}}{4 !}+\frac{\Phi^{2}}{2} \delta_{2}+\frac{\Phi^{4}}{4 !} \delta_{1}+\delta_{3}-\frac{3 b^{4}}{128 \pi^{2} \sqrt{1+\chi}}+\frac{b^{4} \ln \left(\frac{b^{2}}{\mu^{2}}\right)}{64 \pi^{2} \sqrt{1+\chi}} \\
& -\frac{b^{2}}{16 \pi^{2} \sqrt{1+\chi} a^{2}} \sum_{n=1}^{\infty} \frac{\left[K_{2}(4 b a n)-2 K_{2}(2 b a n)\right]}{n^{2}}
\end{aligned}
$$

where the renormalization constants $\delta_{1}, \delta_{2}$, and $\delta_{3}$ need to be determined in order to find the renormalized form for the effective potential [Eq. (2.86)]. For this purpose, the conditions (2.8), (2.9), and (2.11) provide

$$
\begin{gathered}
\frac{\delta_{1}}{4 !}=\frac{\lambda^{2} \ln \left(\frac{\mu^{2}}{m^{2}}\right)}{256 \pi^{2} \sqrt{1+\chi}}, \\
\frac{\delta_{2}}{2}=\frac{\lambda m^{2} \ln \left(\frac{\mu^{2}}{m^{2}}\right)}{64 \pi^{2} \sqrt{1+\chi}}+\frac{\lambda m^{2}}{64 \pi^{2} \sqrt{1+\chi}},
\end{gathered}
$$

and

$$
\delta_{3}=\frac{m^{4} \ln \left(\frac{\mu^{2}}{m^{2}}\right)}{64 \pi^{2} \sqrt{1+\chi}}+\frac{3 m^{4}}{128 \pi^{2} \sqrt{1+\chi}} .
$$

The renormalization constants found above, when used in Eq. (2.86), allow us to obtain the renormalized effective potential up to one-loop correction:

$$
\begin{aligned}
V_{\mathrm{eff}}^{\mathrm{R}}(\Phi)= & \frac{m^{2} \Phi^{2}}{2}+\frac{\lambda \Phi^{4}}{24}-\frac{3 \lambda^{2} \Phi^{4}}{512 \pi^{2} \sqrt{1+\chi}}-\frac{\lambda m^{2} \Phi^{2}}{128 \pi^{2} \sqrt{1+\chi}} \\
& +\frac{\lambda^{2} \Phi^{4} \ln \left(\frac{b^{2}}{m^{2}}\right)}{256 \pi^{2} \sqrt{1+\chi}}+\frac{\lambda m^{2} \Phi^{2} \ln \left(\frac{b^{2}}{m^{2}}\right)}{64 \pi^{2} \sqrt{1+\chi}}+\frac{m^{4} \ln \left(\frac{b^{2}}{m^{2}}\right)}{64 \pi^{2} \sqrt{1+\chi}} \\
& -\frac{b^{2}}{16 \pi^{2} \sqrt{1+\chi} a^{2}} \sum_{n=1}^{\infty} \frac{\left[K_{2}(4 a b n)-2 K_{2}(2 a b n)\right]}{n^{2}} .
\end{aligned}
$$

The renormalized effective potential above, at $\Phi=0$, provides the vacuum energy per unit area of the plates as

$$
\begin{aligned}
\frac{E_{C}}{L^{2}} & =a V_{\mathrm{eff}}^{\mathrm{R}}(0) \\
& =-\frac{m^{2}}{16 \pi^{2} \sqrt{1+\chi} a} \sum_{n=1}^{\infty} \frac{\left[K_{2}(4 a m n)-2 K_{2}(2 a m n)\right]}{n^{2}} .
\end{aligned}
$$

This expression is a convergent and exact expression for the vacuum energy. From it, we can consider asymptotic expressions for small and large arguments of the modified Bessel function $K_{\mu}(x)$.

Let us now show the asymptotic expressions in the regimes $m a \ll 1$ and $m a \gg 1$. In the latter, the vacuum energy (2.91) is exponentially suppressed and dominated by the term $n=1$ of the modified Bessel function in the sum; i.e.,

$$
\frac{E_{C}}{L^{2}} \approx \frac{1}{32 \sqrt{1+\chi}}\left(\frac{m}{\pi a}\right)^{3 / 2} e^{-2 a m} .
$$

On the other hand, in the regime $m a \ll 1$, the vacuum energy is given by

$$
\frac{E_{C}}{L^{2}} \approx \frac{7 \pi^{2}}{11520 \sqrt{1+\chi} a^{3}}-\frac{a m^{4}}{48 \pi^{2} \sqrt{1+\chi}}+\frac{a^{2} m^{5}}{60 \pi^{2} \sqrt{1+\chi}} .
$$

This approximated expression is dominated by the first term on the rhs, associated with the massless scalar field.

Now we turn to the calculation of the two-loop correction to the effective potential. As in the previous sections, we can also make use of the zeta function, which in the present case is given by Eq. (2.83). Thus, the function $S_{1}(\Phi)$ is written in the form

$$
\begin{aligned}
S_{1}(\Phi)= & \left\{\sum _ { n = 0 } ^ { \infty } \frac { 1 } { a } \int \frac { d ^ { 3 } k } { ( 2 \pi ) ^ { 3 } } \left[(1+\chi) k_{t}^{2}+k^{2}\right.\right. \\
& \left.\left.+\left(\left(n+\frac{1}{2}\right) \frac{\pi}{a}\right)^{2}+m^{2}+\frac{\lambda \Phi^{2}}{2}\right]^{-s}\right\}^{2}
\end{aligned}
$$

and can be expressed in terms of the zeta function (2.83) as

$$
S_{1}(\Phi)=\left[\frac{\zeta_{R}(1)}{V_{3} a}\right]^{2}
$$

where $\zeta_{R}(1)$ is the zeta function (2.83) taken at $s=1$ after subtracting the divergent part of it given by the first term on the rhs. As explained before, this divergent part, when divided by $V_{3} a$, does not depend on $a$, and as is customary, must be subtracted. Thus, from Eqs. (2.95) and (2.37), we obtain the two-loop correction to the vacuum energy as

$$
\begin{aligned}
\frac{E_{C}^{(\lambda)}}{L^{2}} & =a V^{(2)}(0) \\
& =\frac{\lambda m^{2}}{128 \pi^{4}(1+\chi) a}\left\{\sum_{n=1}^{\infty} \frac{\left[K_{1}(4 a m n)-K_{1}(2 a m n)\right]}{n}\right\}^{2},
\end{aligned}
$$





FIG. 6. The behavior of the Casimir energy per unit area $E(a m)=\frac{a^{3}}{L^{2}} E_{C}$ given by Eq. (2.91) as a function of $a m$ is exhibited in the left plot. The behavior of the two-loop contribution $E_{\lambda}(a m)=\frac{a^{3}}{L^{2}} E_{C}^{(\lambda)}$, given by Eq. (2.96), is exhibited in the right plot. For the latter, we assume $\lambda=10^{-3}$, and consider different values for the parameter, $\chi$.

which is also a convergent and exact expression. It is also exponentially suppressed for $m a \gg 1$ and provides the massless contribution for $m a \ll 1$.

The exponentially suppressed mathematical expression for Eq. (2.96) in the regime $m a \gg 1$ is given by

$$
\frac{E_{C}^{(\lambda)}}{L^{2}} \approx \frac{\lambda m e^{-4 a m}}{512 \pi^{3}(1+\chi) a^{2}},
$$

while in the regime $m a \ll 1$, the vacuum energy is written as

$$
\begin{aligned}
\frac{E_{C}^{(\lambda)}}{L^{2}} \approx & \frac{\lambda}{73728(1+\chi) a^{3}}-\frac{\lambda m^{2}}{3072 \pi^{2}(1+\chi) a} \\
& +\frac{\lambda m^{3}}{4608 \pi^{2}(1+\chi)},
\end{aligned}
$$

where we can clearly see that the first term on the rhs is the dominant one and is associated with the massless scalar field.

In the left panel of Fig. 6, we exhibit the Casimir energy, given by Eq. (2.91), as a function of am, whereas in the right panel we exhibit the behavior of the two-loop correction to the Casimir energy per unit area, given by Eq. (2.96), as a function of am, considering different values for $\chi$ and fixing $\lambda=10^{-5}$. By these plots, we can infer that the vacuum energy and its two-loop correction decrease as $\chi$ increases. Note that this is different from the timelike case considering only the Dirichlet or Neumann boundary condition, in which case the vacuum energy increases, whereas its radiative correction decreases, as $\chi$ increases.

The mixed boundary condition we are considering here will also generate, at the one-loop level, a topological mass.
In this sense, we can obtain the topological mass by using Eqs. (2.90) and (2.9). It is given by

$m_{\mathrm{T}}^{2}=m^{2}\left\{1+\frac{\lambda}{8 \pi^{2} \sqrt{1+\chi} a m} \sum_{n=1}^{\infty} \frac{\left[K_{1}(4 a m n)-K_{1}(2 a m n)\right]}{n}\right\}$.

We have plotted in Fig. 7 the behavior of $\frac{m_{T}}{m}$ by using Eq. (2.99) in terms of am. The plot shows that the topological mass increases as the Lorentz symmetry violation parameter, $\chi$, increases. This is different from the timelike case considering only the Dirichlet or Neumann boundary condition, in which case the topological mass decreases as $\chi$ increases. Figure 7 also shows that in the regime $m a \gg 1$, the topological mass is dominated by the first term on the rhs of Eq. (2.99), while in the opposite limit $m a \ll 1$, the topological mass is the one associated with a massless scalar field.

Mathematically, for $m a \gg 1$, we have

$$
m_{\mathrm{T}}^{2} \approx m^{2}-\frac{\lambda}{16 \sqrt{1+\chi}} \frac{\sqrt{m} e^{-2 a m}}{(\pi a)^{3 / 2}},
$$

which shows that the topological mass is dominated by the first term, $m^{2}$.

In the opposite regime, $m a \ll 1$, we obtain

$$
\begin{aligned}
m_{\mathrm{T}}^{2} \approx & m^{2}-\frac{\lambda}{192 \sqrt{1+\chi} a^{2}}+\frac{\lambda m^{2}}{16 \pi^{2} \sqrt{1+\chi}}-\frac{\lambda a m^{3}}{24 \pi^{2} \sqrt{1+\chi}} \\
& +\frac{\lambda a^{3} m^{5}}{120 \pi^{2} \sqrt{1+\chi}} .
\end{aligned}
$$




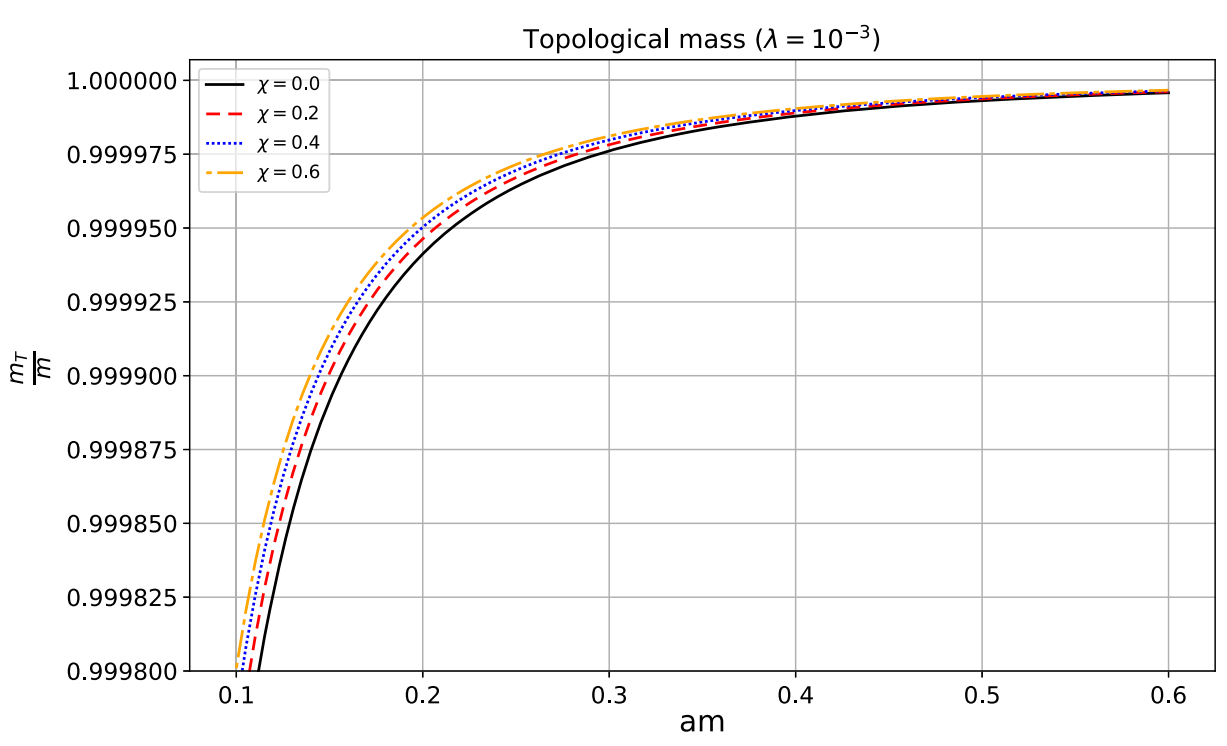

FIG. 7. The ratio of the topological mass to the field mass as a function of $\mathrm{am}$. The plot considers $\lambda=10^{-3}$ and different values of $\chi$.

In this limit, the dominant term is the second one on the rhs of the above approximation, associated with a massless scalar field.

\section{Spacelike vector}

We want now to consider the case in which the constant four-vector, $u^{\mu}$, is of the spacelike type. As before, there are three spacelike components specifying the broken symmetry direction: $u^{x}=(0,1,0,0), u^{y}=(0,0,1,0)$, both parallel to the plates, and $u^{z}=(0,0,0,1)$, orthogonal to the plates. In the two first cases, specifying the $x$ and $y$ directions, parallel to the plates, the results for the effective potential, Casimir energy, and topological mass are the same. Let us then consider the $x$ direction:

$$
u^{x}=(0,1,0,0) \text {. }
$$

In this case, from Eq. (2.77), the set of eigenvalues is given by

$\Lambda_{\beta}=k^{2}+(1-\chi) k_{x}^{2}+\left[\left(n+\frac{1}{2}\right) \frac{\pi}{a}\right]^{2}+m^{2}+\frac{\lambda \Phi^{2}}{2}$,

where $k^{2}=k_{t}^{2}+k_{y}^{2}$. So, using Eq. (2.6), the zeta function is written as

$$
\zeta(s)=\frac{V_{3}}{(2 \pi)^{3}} \int d^{3} k \sum_{n=1}^{\infty}\left\{k^{2}+(1-\chi) k_{x}^{2}+\left[\left(n+\frac{1}{2}\right) \frac{\pi}{a}\right]^{2}+m^{2}+\frac{\lambda \Phi^{2}}{2}\right\}^{-s} .
$$

Again, by using the identity (2.16), we are able to solve the integrals in $k_{t}, k_{x}$, and $k_{y}$ to obtain the zeta function in the form

$$
\zeta(s)=\frac{1}{\sqrt{1-\chi}} \frac{V_{3}}{(2 \pi)^{3}} \frac{\pi^{3 / 2} \Gamma(s-3 / 2)}{\Gamma(s)} w^{3-2 s} \sum_{n=0}^{\infty}\left[\left(n+\frac{1}{2}\right)^{2}+\nu^{2}\right]^{3 / 2-s},
$$

where

$$
\nu^{2}=\frac{\lambda \Phi^{2}}{2 w^{2}}+\frac{m^{2}}{w^{2}} \quad \text { and } \quad w=\frac{\pi}{a} .
$$

We can notice that the expression (2.105) can be obtained from Eq. (2.80) by making $\chi \rightarrow-\chi$. Consequently, all the results for the renormalized effective potential, Casimir energy, and topological mass can be obtained from the timelike case considered previously. This is also valid if we consider the vector $u^{y}$.
The most important type of Lorentz symmetry violation in this section is one occurring in the orthogonal direction to the plates; that is,

$$
u^{z}=(0,0,0,1)
$$

In this case, the set of eigenvalues (2.77) becomes

$$
\Lambda_{\beta}=k^{2}+(1-\chi)\left[\left(n+\frac{1}{2}\right) \frac{\pi}{a}\right]^{2}+m^{2}+\frac{\lambda \Phi^{2}}{2},
$$


where $k^{2}=k_{t}^{2}+k_{x}^{2}+k_{y}^{2}$. Thus, substituting Eq. (2.108) into Eq. (2.6), we have the zeta function expression written as

$$
\zeta(s)=\frac{V_{3}}{(2 \pi)^{3}} \sum_{n=0}^{\infty} \int d^{3} k\left\{k^{2}+(1-\chi)\left[\left(n+\frac{1}{2}\right) \frac{\pi}{a}\right]^{2}+m^{2}+\frac{\lambda \Phi^{2}}{2}\right\}^{-s} .
$$

Once again, by using Eq. (2.16), we obtain

$$
\zeta(s)=\frac{V_{3}}{(2 \pi)^{3}} \frac{\pi^{3 / 2} \Gamma(s-3 / 2)}{\Gamma(s)} w^{3-2 s} \sum_{n=0}^{\infty}\left[\left(n+\frac{1}{2}\right)^{2}+\nu^{2}\right]^{3 / 2-s},
$$

where

$$
\nu^{2} \equiv \frac{\lambda \Phi^{2}}{2 w^{2}}+\frac{m^{2}}{w^{2}} \quad \text { and } \quad w \equiv \sqrt{1-\chi} \frac{\pi}{a}
$$

We need now to find an expression for the sum in $n$ present in the zeta function above. In order to do that, we can make use of Eq. (2.82). This provides

$$
\zeta(s)=\frac{V_{3}}{(2 \pi)^{3}} \frac{\pi^{3 / 2} \Gamma(\bar{s})}{\Gamma(\bar{s}+3 / 2)}\left(\frac{w}{2}\right)^{-2 \bar{s}}\left[\sum_{n=1}^{\infty}\left(n^{2}+4 \nu^{2}\right)^{-\bar{s}}-2^{-2 \bar{s}} \sum_{n=1}^{\infty}\left(n^{2}+\nu^{2}\right)^{-\bar{s}}\right],
$$

where we have $\bar{s}=s-3 / 2$. Hence, the Epstein-Hurwitz zeta function (2.19) allows us to obtain Eq. (2.112) as

$$
\begin{aligned}
\zeta(s)= & \frac{V_{3}}{(2 \pi)^{3}}\left\{\frac{\pi^{2} w^{-2 \bar{s}} \nu^{1-2 \bar{s}} \Gamma(\bar{s}-1 / 2)}{2 \Gamma(\bar{s}+3 / 2)}+\frac{2^{\bar{s}+1 / 2} \pi^{2 \bar{s}+1} w^{-2 \bar{s}}}{\Gamma(\bar{s}+3 / 2)}\right. \\
& \left.\times\left[4^{\bar{s}} \sum_{n=1}^{\infty} n^{2 \bar{s}-1} f_{\bar{s}-1 / 2}(4 \pi \nu n)-\sum_{n=1}^{\infty} n^{2 \bar{s}-1} f_{\bar{s}-1 / 2}(2 \pi \nu n)\right]\right\} .
\end{aligned}
$$

Consequently, in the limit $s \rightarrow 0$, we have

$$
\zeta(0)=\frac{V_{3} a}{2(2 \pi)^{4}} \frac{\pi^{2} b^{4}}{\sqrt{1-\chi}}
$$

and

$$
\zeta^{\prime}(0)=\frac{V_{3} a}{(2 \pi)^{3}}\left\{\frac{3 \pi b^{4}}{8 \sqrt{1-\chi}}-\frac{\pi b^{4} \ln (b)}{2 \sqrt{1-\chi}}+\frac{\pi b^{2} \sqrt{1-\chi}}{a^{2}} \sum_{n=1}^{\infty} \frac{\left[K_{2}\left(\frac{4 a b n}{\sqrt{1-\chi}}\right)-2 K_{2}\left(\frac{2 a b n}{\sqrt{1-\chi}}\right)\right]}{n^{2}}\right\}
$$

The one-loop correction (2.5) to the effective potential is now possible to obtain by using the results above for $\zeta(0)$ and $\zeta^{\prime}(0)$. This gives

$$
V^{(1)}(\Phi)=\frac{b^{4} \ln \left(\frac{b^{2}}{\mu^{2}}\right)}{64 \pi^{2} \sqrt{1-\chi}}-\frac{3 b^{4}}{128 \pi^{2} \sqrt{1-\chi}}-\frac{b^{2} \sqrt{1-\chi}}{16 \pi^{2} a^{2}} \sum_{n=1}^{\infty} \frac{\left[K_{2}\left(\frac{4 a b n}{\sqrt{1-\chi}}\right)-2 K_{2}\left(\frac{2 a b n}{\sqrt{1-\chi}}\right)\right]}{n^{2}}
$$

where

$$
b=\sqrt{\frac{\lambda \Phi^{2}}{2}+m^{2}}
$$

Furthermore, the effective potential up to one-loop correction, from Eqs. (2.3) and (2.116), is written as

$$
\begin{aligned}
V_{\mathrm{eff}}(\Phi)= & \frac{m^{2} \Phi^{2}}{2}+\frac{\lambda \Phi^{4}}{4 !}+\frac{\Phi^{2}}{2} \delta_{2}+\frac{\Phi^{4}}{4 !} \delta_{1}+\delta_{3}+\frac{b^{4} \ln \left(\frac{b^{2}}{\mu^{2}}\right)}{64 \pi^{2} \sqrt{1-\chi}}-\frac{3 b^{4}}{128 \pi^{2} \sqrt{1-\chi}} \\
& -\frac{b^{2} \sqrt{1-\chi}}{16 \pi^{2} a^{2}} \sum_{n=1}^{\infty} \frac{\left[K_{2}\left(\frac{4 a b n}{\sqrt{1-\chi}}\right)-2 K_{2}\left(\frac{2 a b n}{\sqrt{1-\chi}}\right)\right]}{n^{2}}
\end{aligned}
$$


where the renormalization constants $\delta_{1}, \delta_{2}$, and $\delta_{3}$ are to be found by using Eq. (2.118) and the conditions given by Eqs. (2.8), (2.9), and (2.11). This provides

$$
\begin{gathered}
\frac{\delta_{1}}{4 !}=\frac{\lambda^{2} \ln \left(\frac{\mu^{2}}{m^{2}}\right)}{256 \pi^{2} \sqrt{1-\chi}}, \\
\frac{\delta_{2}}{2}=\frac{\lambda m^{2} \ln \left(\frac{\mu^{2}}{m^{2}}\right)}{64 \pi^{2} \sqrt{1-\chi}}+\frac{\lambda m^{2}}{64 \pi^{2} \sqrt{1-\chi}}
\end{gathered}
$$

and

$$
\delta_{3}=\frac{m^{4} \ln \left(\frac{\mu^{2}}{m^{2}}\right)}{64 \pi^{2} \sqrt{1-\chi}}+\frac{3 m^{4}}{128 \pi^{2} \sqrt{1-\chi}} .
$$

Finally, by using the renormalization constants found above in Eq. (2.118), we obtain the renormalized effective potential up to one-loop correction; i.e.,

$$
\begin{aligned}
V_{\mathrm{eff}}^{\mathrm{R}}(\Phi)= & \frac{m^{2} \Phi^{2}}{2}+\frac{\lambda \Phi^{4}}{24}-\frac{\lambda m^{2} \Phi^{2}}{128 \pi^{2} \sqrt{1-\chi}}-\frac{3 \lambda^{2} \Phi^{4}}{512 \pi^{2} \sqrt{1-\chi}} \\
& +\frac{\lambda m^{2} \Phi^{2} \ln \left(\frac{b^{2}}{m^{2}}\right)}{64 \pi^{2} \sqrt{1-\chi}}+\frac{m^{4} \ln \left(\frac{b^{2}}{m^{2}}\right)}{64 \pi^{2} \sqrt{1-\chi}}+\frac{\lambda^{2} \Phi^{4} \ln \left(\frac{b^{2}}{m^{2}}\right)}{256 \pi^{2} \sqrt{1-\chi}} \\
& -\frac{b^{2} \sqrt{1-\chi}}{16 \pi^{2} a^{2}} \sum_{n=1}^{\infty} \frac{\left[K_{2}\left(\frac{4 a b n}{\sqrt{1-\chi}}\right)-2 K_{2}\left(\frac{2 a b n}{\sqrt{1-\chi}}\right)\right]}{n^{2}} .
\end{aligned}
$$

At this point, we can, by using the renormalized effective potential (2.122), obtain the vacuum energy per unit area of the plate. This is done by taking Eq. (2.122) at $\Phi=0$. This gives

$\frac{E_{C}}{L^{2}}=a V_{\mathrm{eff}}^{\mathrm{R}}(0)=-\frac{m^{2} \sqrt{1-\chi}}{16 \pi^{2} a} \sum_{n=1}^{\infty} \frac{\left[K_{2}\left(\frac{4 a m n}{\sqrt{1-\chi}}\right)-2 K_{2}\left(\frac{2 a m n}{\sqrt{1-\chi}}\right)\right]}{n^{2}}$.

This exact and closed expression for the vacuum energy is exponentially suppressed for $m a \gg 1$, while for $m a \ll 1$ it provides the expression for the vacuum energy in the massless scalar field case.

The exponentially suppressed expression for the vacuum energy in the regime $m a \gg 1$ is dominated by the $n=1$ term of the sum, providing

$$
\frac{E_{C}}{L^{2}} \approx \frac{(1-\chi)^{3 / 4}}{16}\left(\frac{m}{\pi a}\right)^{3 / 2} e^{-\frac{2 a m}{\sqrt{1-\chi}}}
$$

The opposite regime, $m a \ll 1$, provides the approximated expression for the vacuum energy

$$
\frac{E_{C}}{L^{2}} \approx \frac{7 \pi^{2}(1-\chi)^{3 / 2}}{11520 a^{3}}-\frac{a m^{4}}{48 \pi^{2} \sqrt{1-\chi}}+\frac{a^{2} m^{5}}{60 \pi^{2}(1-\chi)},
$$

which is dominated by the first term on the rhs. This is the term associated with the vacuum energy of the massless scalar field.

We can now turn the calculation of the two-loop correction to the effective potential at $\Phi=0$. The $S_{1}(\Phi)$ function, as before, is given by

$$
\begin{aligned}
S_{1}(\Phi)= & \left\{\sum _ { n = 0 } ^ { \infty } \frac { 1 } { a } \int \frac { d ^ { 3 } k } { ( 2 \pi ) ^ { 3 } } \left[k^{2}+(1-\chi)\left[\left(n+\frac{1}{2}\right) \frac{\pi}{a}\right]^{2}\right.\right. \\
& \left.\left.+m^{2}+\frac{\lambda \Phi^{2}}{2}\right]^{-s}\right\}^{2},
\end{aligned}
$$

which can be expressed in terms of the zeta function (2.113) as

$$
S_{1}(\Phi)=\left[\frac{\zeta_{R}(1)}{V_{3} a}\right]^{2}
$$

where $\zeta_{R}(1)$ is the zeta function (2.83) taken at $s=1$ after subtracting the divergent part of it given by the first term on the rhs. Again, this divergent part, when divided by $V_{3} a$, does not depend on $a$ and, as customary, must be subtracted. Thus, from Eqs. (2.127) and (2.37), we obtain the two-loop correction to the vacuum energy as

$$
\frac{E_{C}^{(\lambda)}}{L^{2}}=a V^{(2)}(0)=\frac{\lambda m^{2}}{128 \pi^{4} a}\left\{\sum_{n=1}^{\infty} \frac{\left[K_{1}\left(\frac{4 a m n}{\sqrt{1-\chi}}\right)-K_{1}\left(\frac{2 a m n}{\sqrt{1-\chi}}\right)\right]}{n}\right\}^{2} .
$$

This is an exact and convergent expression for the correction of the vacuum energy per unit area. The asymptotic behaviors for the above expression are explicitly provided below.

The exponentially suppressed expression for the vacuum energy correction in the regime $m a \gg 1$ is dominated by the $n=1$ term:

$$
\frac{E_{C}^{(\lambda)}}{L^{2}} \approx \frac{\lambda m \sqrt{1-\chi} e^{-\frac{4 a m}{\sqrt{1-\chi}}}}{512 \pi^{3} a^{2}} .
$$

On the other hand, the expression for the vacuum energy correction in the opposite regime $m a \ll 1$ is given by

$$
\begin{aligned}
\frac{E_{C}^{(\lambda)}}{L^{2}} \approx & \frac{\lambda(1-\chi)}{73728 a^{3}}-\frac{\lambda m^{2}}{3072 \pi^{2} a}+\frac{\lambda m^{3}}{4608 \pi^{2} \sqrt{1-\chi}} \\
& +\frac{\lambda a m^{4}}{512 \pi^{4}(1-\chi)} .
\end{aligned}
$$



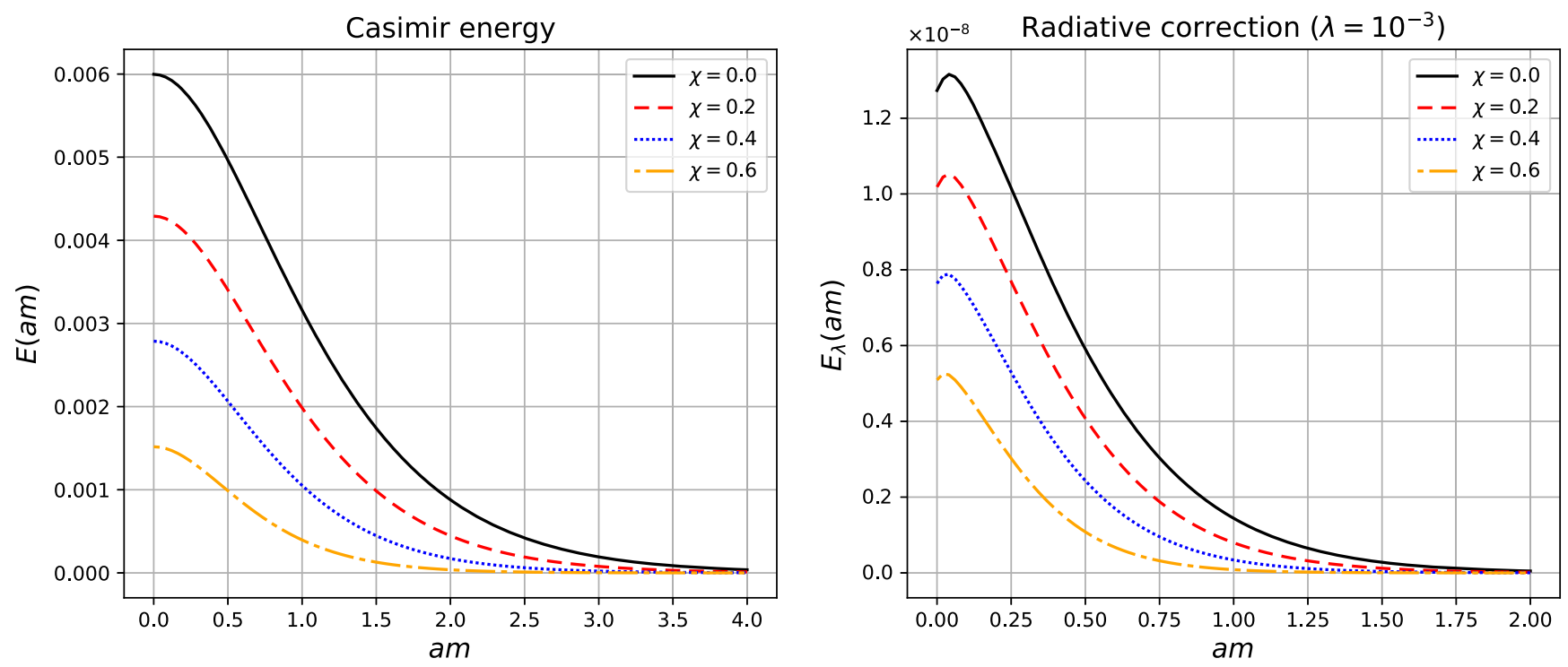

FIG. 8. The left plot presents the behavior of Casimir energy per unit area $E(a m)=\frac{a^{3}}{L^{2}} E_{C}$, given by Eq. (2.123), as a function of $a m$. The two-loop contribution $E_{\lambda}(\mathrm{am})=\frac{a^{3}}{L^{2}} E_{C}^{(\lambda)}$, given by Eq. (2.128), is exhibited in the right panel as a function of $a m$, fixing $\lambda=10^{-3}$. In both plots, we have considered various values of $\chi$.

This expression is dominated by the first term on the rhs and is associated with the vacuum energy correction in the massless scalar field case.

In the left panel of Fig. 8, we exhibit the behavior of the Casimir energy, Eq. (2.123), as a function of am. In the right panel is exhibited the vacuum energy radiative correction, Eq. (2.128), also as a function of am. In both cases, the energy values decreases as $\chi$ increases. The curves are shifted down more than in the timelike case. This is due to the dependence of the vacuum energy (2.123) and its radiative correction (2.128) on $\chi$, in the argument of the modified Bessel function, $K_{\mu}(x)$. The vacuum energy (2.123) also depends on $\chi$ as a multiplicative factor.

We want now to analyze the generation of topological mass. The latter can be obtained by using the renormalized effective potential (2.122) in the condition (2.9). This provides the exact expression for the topological mass; that is,

$$
m_{\mathrm{T}}^{2}=m^{2}\left\{1+\frac{\lambda}{8 \pi^{2} a m} \sum_{n=1}^{\infty} \frac{\left[K_{1}\left(\frac{4 a m n}{\sqrt{1-\chi}}\right)-K_{1}\left(\frac{2 a m n}{\sqrt{1-\chi}}\right)\right]}{n}\right\} .
$$

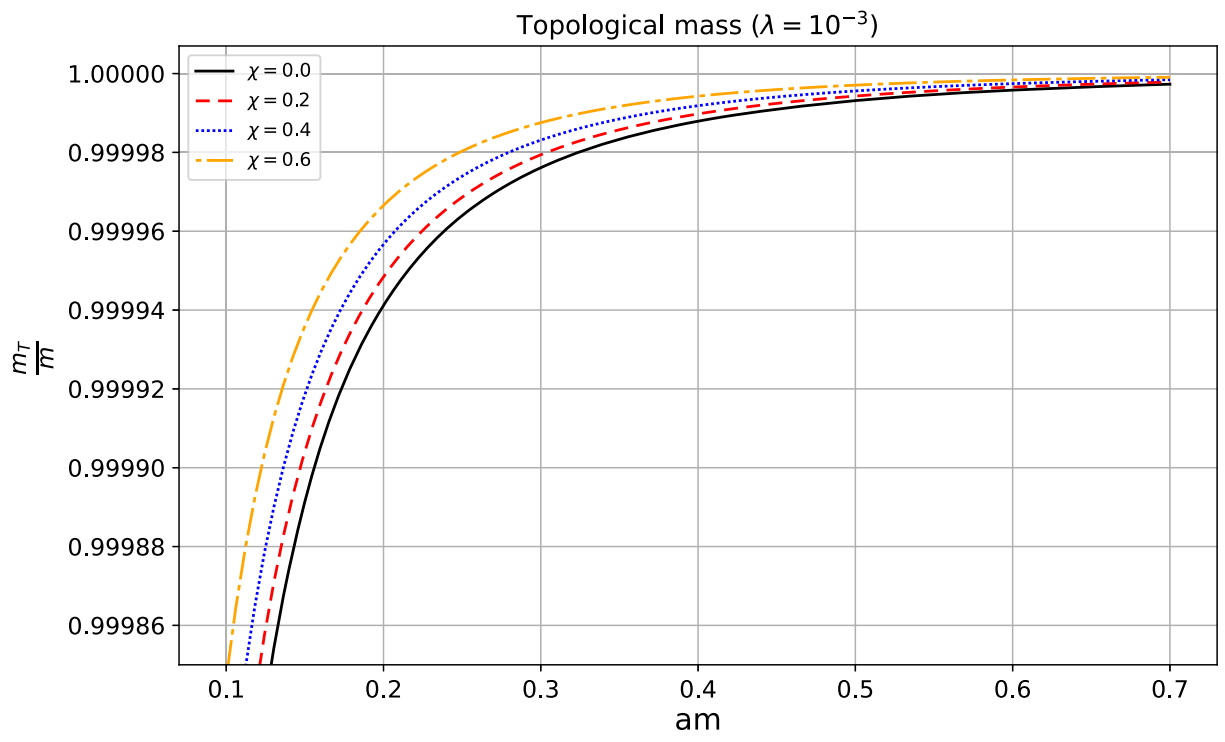

FIG. 9. Graph presenting behavior of the ratio of the topological mass to the mass of the field as a function of $a m$. The plot considers $\lambda=10^{-3}$ and different values for the Lorentz-violating parameter, $\chi$. 
This expression is plotted in Fig. 9. We can see that in the regime $m a \gg 1$, the topological mass is dominated by the first term on the rhs and grows to infinity. In the opposite regime $m a \ll 1$, the topological mass tends to the expression associated with the massless scalar field. We can also see in Fig. 9 that the topological mass increases as $\chi$ increases. The curves are shifted up more than in the previous timelike case as a consequence of the dependence of the topological mass [Eq. (2.131)] on $\chi$, in the argument of the modified Bessel function.

The topological mass (2.131) in the regime $m a \gg 1$ is given by

$$
m_{\mathrm{T}}^{2} \approx m^{2}-\frac{\lambda \sqrt[4]{1-\chi}}{16 \pi^{3 / 2}} \sqrt{\frac{m}{a^{3}}} e^{-\frac{2 a m}{\sqrt{1-\chi}}},
$$

while in the opposite regime, $m a \ll 1$, it is

$$
\begin{aligned}
m_{\mathrm{T}}^{2} \approx & m^{2}-\frac{\lambda \sqrt{1-\chi}}{192 a^{2}}-\frac{\lambda m^{2}}{16 \pi^{2} \sqrt{1-\chi}}-\frac{\lambda a m^{3}}{24 \pi^{2}(1-\chi)} \\
& +\frac{\lambda a^{3} m^{5}}{120 \pi^{2}(1-\chi)^{2}} .
\end{aligned}
$$

Note that the second term on the rhs of Eq. (2.133) is associated with a massless scalar field.

\section{CONCLUDING REMARKS}

In this work, we have investigated the Casimir effect and the generation of topological mass associated with a scalar, self-interacting $\lambda \phi^{4}$ field theory in the context of an aethertype Lorentz symmetry violation model, implemented by direct coupling between the derivative of the field with an external constant four-vector. Specifically, we have considered the situation in which the field is confined between two parallel plates, assuming that it obeys, on each of the plates, Dirichlet, Newman and mixed boundary conditions, separately. The area of the plates has been taken to be $L^{2}$, whereas the distance between them has been taken as $a(a \ll L)$.

Furthermore, we have found exactly $\Phi$-dependent renormalized effective potentials, up to one-loop correction, considering both timelike and spacelike cases of the fourvector $u^{\mu}$, where $\Phi$ is the classical and fixed background field. These renormalized effective potentials, at $\Phi=0$, provided a Casimir-like energy and topological mass for all cases. We have also obtained an exact two-loop correction to the effective potential when $\Phi=0$, which allows us to find a radiative correction to the Casimir-like energy obtained from the renormalized effective potential up to one-loop correction. The Casimir-like energies from Dirichlet and Neumann boundary conditions are equal and differ from the Casimir-like energy arising from the mixed boundary condition by a numerical factor and also by a change of sign.
The Casimir-like effect and its radiative correction, as well as the topological mass, depend upon specific boundary conditions imposed on the fields and the Lorentzsymmetry-breaking parameter, $\chi$. It is worth pointing out that in all boundary condition cases considered, our results are more affected by $\chi$ in the spacelike type of broken symmetry-specifically, in the $z$ direction, orthogonal to the plates. Note that the results obtained here considering Dirichlet, Neumann, and mixed boundary conditions at one-loop level agree with well-known results in the case where the Lorentz symmetry is preserved-that is, $\chi=0$ $[41,42]$. This is also true at two-loop levels-i.e., we also recover, in the massless scalar field case, the expressions obtained for the Casimir energy density and topological mass in Ref. [30], and the Casimir energy, considering the three boundary conditions, in the massless field limit, given in Refs. [43,44]. These last three papers used the Riemann zeta-function renormalization to obtain the Casimir energy. The two-loop correction to the Casimir energy associated with the scalar field under the Dirichlet boundary condition, in the absence of Lorentz symmetry violation, was also calculated in Ref. [45], using the box renormalization scheme (BRS). However, in the latter, the Casimir energy correction in the massless limit disagrees with the results found in Refs. $[30,43,44]$ by a negative sign. The reason for this is the convention adopted in the definition of the twoloop correction in Ref. [45], which presents a minus sign in the expression analogue to Eq. (2.37) of our present paper. Consequently, the total Casimir energy will be decreased. From the physical point of view, it is expected that the scalar self-interaction would increase the Casimir energy, and not the opposite. Our results are in accordance with this assumption.

The analysis for the Casimir energy density considering an aether-type Lorentz symmetry violation term in the case of a Dirichlet self-interacting scalar field has also been considered in Ref. [46]. There the authors have obtained the first-order radiative correction in $\lambda$ to the Casimir energy density by using BRS. Moreover, the definition adopted to evaluate this correction is the same as that given in Ref. [45]; consequently, a negative contribution to the Casimir energy is obtained. Finally, we want to emphasize that in our present paper we have considered, besides the Dirichlet boundary condition, also the Neumann and mixed ones to obtain the first-order correction in $\lambda$ to the Casimir energy. Furthermore, we have used a different renormalization method based on the zeta function. We have also shown that in each one of the boundary conditions considered, a topological mass is generated.

Let us now, before ending the conclusions, discuss implications and the possibility of observational detection of a violation in the Lorentz symmetry in light of our results. As is known, the energy scale where the Lorentz symmetry is expected to be broken is of the order of the Planck scale, something around $10^{19} \mathrm{GeV}$. This makes it difficult in principle to envisage an experiment capable of 
detecting signals of Lorentz symmetry violation. Nevertheless, a Casimir energy density analysis considering models of Lorentz symmetry violation, such as the one considered here, can offer a possible way of detecting signals in low-energy scales. In particular, one can consider, for instance, extensions of the Standard Model of particle physics where violations in the Lorentz symmetry are taken into consideration. In these scenarios, looking at the Higgs sector where a beta decay is observed, a bound of $\chi<10^{-6}$ is obtained. Also, a bound of $\chi<10^{-19}$ is obtained considering lasers based on interferometry [15]. If these bounds are used in our results, finite values could be obtained and experiments for the detection of the Casimir energy could confirm the theoretical results. Likewise, if the detailed observations and measurements for the
Casimir-like effect were possible, one could use the modifications of it by the Lorentz symmetry violation model considered here to estimate the values of the parameter $\chi$ describing the spacetime anisotropy. This would certainly contribute to the experimental measurement attempts to get an upper bound on $\chi$.

\section{ACKNOWLEDGMENTS}

M. B.C. is supported by Conselho Nacional de Desenvolvimento Científico e Tecnológico-Brasil (CNPq) through Project No. 150479/2019-0. E. R. B. M. is partially supported by CNPq under Grant No. 301.783/ 2019-3 . H. F. S. M. is partially supported by CNPq under Grants No. 305379/2017-8 and No. 430002/2018-1.
[1] H. Casimir, Indagat. Math 10, 261 (1948).

[2] M. Sparnaay, Physica (Utrecht) 24, 751 (1958).

[3] G. Bressi, G. Carugno, R. Onofrio, and G. Ruoso, Phys. Rev. Lett. 88, 041804 (2002).

[4] S. Lamoreaux, Phys. Rev. Lett. 78, 5 (1997).

[5] S. K. Lamoreaux, Phys. Rev. Lett. 78, 5 (1997); 81, 5475(E) (1998).

[6] U. Mohideen and A. Roy, Phys. Rev. Lett. 81, 4549 (1998).

[7] V. M. Mostepanenko, Braz. J. Phys. 30, 309 (2000).

[8] W. J. Kim, M. Brown-Hayes, D. A. R. Dalvit, J. H. Brownell, and R. Onofrio, Phys. Rev. A 78, 020101 (2008).

[9] Q. Wei, D. A. R. Dalvit, F. C. Lombardo, F. D. Mazzitelli, and R. Onofrio, Phys. Rev. A 81, 052115 (2010).

[10] V. Kostelecky and S. Samuel, Phys. Rev. D 39, 683 (1989).

[11] A. Anisimov, T. Banks, M. Dine, and M. Graesser, Phys. Rev. D 65, 085032 (2002).

[12] C. E. Carlson, C. D. Carone, and R. F. Lebed, Phys. Lett. B 518, 201 (2001).

[13] J. L. Hewett, F. J. Petriello, and T. G. Rizzo, Phys. Rev. D 64, 075012 (2001).

[14] O. Bertolami and L. Guisado, J. High Energy Phys. 12 (2003) 013.

[15] V. Kostelecky, R. Lehnert, and M. J. Perry, Phys. Rev. D 68, 123511 (2003).

[16] L. Anchordoqui and H. Goldberg, Phys. Rev. D 68, 083513 (2003).

[17] O. Bertolami, Classical Quantum Gravity 14, 2785 (1997).

[18] J. Alfaro, H. A. Morales-Tecotl, and L. F. Urrutia, Phys. Rev. Lett. 84, 2318 (2000).

[19] J. Alfaro, H. A. Morales-Tecotl, and L. F. Urrutia, Phys. Rev. D 65, 103509 (2002).

[20] R. K. Obousy and G. Cleaver, Mod. Phys. Lett. A 24, 1495 (2009).

[21] R. Obousy and G. Cleaver, J. Geom. Phys. 61, 577 (2011).
[22] A. Martín-Ruiz and C. Escobar, Phys. Rev. D 94, 076010 (2016).

[23] A. Martín-Ruiz and C. Escobar, Phys. Rev. D 95, 036011 (2017).

[24] C. Escobar, L. Medel, and A. Martín-Ruiz, Phys. Rev. D 101, 095011 (2020).

[25] R. Jackiw, Phys. Rev. D 9, 1686 (1974).

[26] E. Wieczorek, D. Robaschik, and K. Scharnhorst, Sov. J. Nucl. Phys. 44, 665 (1986).

[27] F. Barone, R. Cavalcanti, and C. Farina, Nucl. Phys. B, Proc. Suppl. 127, 118 (2004).

[28] D. Robaschik, K. Scharnhorst, and E. Wieczorek, Ann. Phys. (N.Y.) 174, 401 (1987).

[29] D. J. Toms, Phys. Rev. D 21, 928 (1980).

[30] D. J. Toms, Phys. Rev. D 21, 2805 (1980).

[31] D. J. Toms, Ann. Phys. (N.Y.) 129, 334 (1980).

[32] S. M. Carroll and H. Tam, Phys. Rev. D 78, 044047 (2008).

[33] A. Chatrabhuti, P. Patcharamaneepakorn, and P. Wongjun, J. High Energy Phys. 08 (2009) 019.

[34] M. Gomes, J. Nascimento, A. Petrov, and A. da Silva, Phys. Rev. D 81, 045018 (2010).

[35] S. R. Coleman and E. J. Weinberg, Phys. Rev. D 7, 1888 (1973).

[36] M. Cruz, E. R. Bezerra de Mello, and A. Y. Petrov, Phys. Rev. D 96, 045019 (2017).

[37] I. Gradstein and I. Ryzhik, Table of Integrals, Series, and Products (Academic Press, New York, 2014).

[38] J. Chung and B. Chung, arXiv:hep-th/9804079.

[39] M. Abramowitz and I. A. Stegun, Handbook of Mathematical Functions with Formulas, Graphs, and Mathematical Tables (Dover Publications, New York, 1972).

[40] M. Bordag, U. Mohideen, and V. Mostepanenko, Phys. Rep. 353, 1 (2001).

[41] K. A. Milton, The Casimir Effect: Physical Manifestations of Zero-Point Energy (World Scientific, River Edge, 2001), p. 301. 
[42] M. Bordag, G. L. Klimchitskaya, U. Mohideen, and V. M. Mostepanenko, Advances in the Casimir Effect, International Series of Monographs on Physics Vol. 145 (Oxford University Press, Oxford, 2009).

[43] F. A. Baron, R. M. Cavalcanti, and C. Farina, Nucl. Phys. B, Proc. Suppl. 127, 118 (2004).
[44] F. A. Barone, R. M. Cavalcanti, and C. Farina, arXiv:hep-th/ 0312169.

[45] R. Moazzemi, M. Namdar, and S. S. Gousheh, J. High Energy Phys. 09 (2007) 029.

[46] A. Mojavezi, R. Moazzemi, and M. E. Zomorrodian, Nucl. Phys. B941, 145 (2019). 